\title{
Gravitational instantons with faster than quadratic curvature decay. I
}

\author{
by \\ Gao Chen \\ University of Science and Technology of China \\ Hefei, China
}

\section{Contents}

1. Introduction . . . . . . . . . . . . . . . . . . 264

Acknowledgement . . . . . . . . . . . . . . . . . 267

2. Notation and definitions . . . . . . . . . . . . . . . 267

3. Asymptotic fibration . . . . . . . . . . . . . . 268

3.1. Short geodesic loops and the local covering space . . . . . . 269

3.2. Control of holonomy of geodesic loops . . . . . . . . . . 273

3.3. Classification of tangent cone at infinity . . . . . . . . . 276

3.4. Decomposing geodesic loops into basis . . . . . . . . . . 279

3.5. From geodesic loops to Riemannian fiberation . . . . . . . . 283

4. The construction of holomorphic functions . . . . . . . . 288

4.1. Weighted Hilbert space . . . . . . . . . . . . . . . . 289

4.2. Elliptic estimates with polynomial growth weights . . . . . 291

4.3. Holomorphic functions on ALF and ALG instantons . . . . . 295

4.4. Exponential decay of curvature of ALH instantons . . . . . 297

4.5. Elliptic estimates with exponential growth weights . . . . . 299

4.6. Holomorphic functions on ALH instantons . . . . . . . . 300

4.7. Compactification of ALG and ALH non-splitting instantons . 302

4.8. Twistor space of ALF- $D_{k}$ instantons . . . . . . . . . . 303

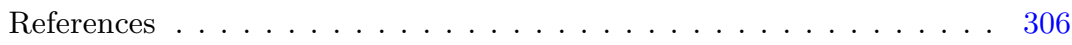




\section{Introduction}

This is our first paper in a series studying gravitational instantons. In 1977, the concept of "gravitational instanton" was first introduced by Hawking as a building block of the Euclidean quantum gravity theory [21]. The literature has provided many different definitions. For clarity, we define a gravitational instanton as a complete, hyperkähler 4-manifold satisfying a decay condition

$$
|\operatorname{Rm}|(x) \leqslant r(x)^{-2-\epsilon},
$$

where $r(x)$ denotes the metric distance to a base point $o$ in the complex surface and $\epsilon>0$ is any small positive number, say $<\frac{1}{100}$. Under those conditions, we want to study two fundamental questions:

(1) What are the differential and metric structures of the infinity of these gravitational instantons? Note that this is different from the tangent cone at infinity, especially if the volume growth is sub-Euclidean.

(2) Given these end structures, to what extent do we know these instantons globally and holomorphically? In other words, is a gravitational instanton uniquely determined by its end structure?

Both problems seem to be well known to the research community. Since 1977, many examples of gravitational instantons have been constructed [21], [2], [29], [12]. The end structures of these examples are completely known now. According to the volume growth rate, they can be divided into four categories: ALE, ALF, ALG, and ALH, where the volume growth is of order $4,3,2$, and 1 , respectively. For readers' convenience, we will give a precise definition of these ends in $\S 2$. There is a folklore conjecture that when the curvature decay is quick enough, any gravitational instanton must be asymptotic to one of the standard models of ends.

In the ALE case, we understand these instantons completely through the works of Kronheimer in [29], [30]. In the remaining cases, the asymptotical volume growth rate is usually difficult to control, may oscillate, and may even not be an integer. In an important paper, with additional assumption that the volume growth rate is sub-Euclidean but at least cubic and a slightly weaker curvature decay condition depending on the volume growth rate, Minerbe [33], [34] proved that it must be ALF. In our paper, we first prove the folklore conjecture.

THeOREM 1.1. (Main Theorem 1) Any connected complete hyperkähler manifold $\left(M^{4}, g\right)$ with curvature decaying as (1.1) must be asymptotic to the standard metric of order $\epsilon$. Consequently, it must be one of the four families: ALE, ALF, ALG, and ALH.

For more detail about this theorem, see Theorems 3.7, 3.11, and 3.19. We would like to remark that the curvature condition cannot be weakened to $|\mathrm{Rm}|=O\left(r^{-2}\right)$. In 
2012, besides the study of ALG and ALH instantons on rational elliptic surfaces, Hein [22] also constructed two new classes of hyperkähler metrics on rational elliptic surfaces with volume growth, injective radius decay, and curvature decay rates $r^{4 / 3}, r^{-1 / 3}, r^{-2}$, and $r^{2},(\log r)^{-1 / 2}, r^{-2}(\log r)^{-1}$, respectively. Note that curvature does not satisfy (1.1) and that they do not belong to any of the four families!

Our new contribution lies in the ALG and ALH cases; in the ALF case, our contribution is to remove the volume growth constraint from Minerbe's work [33]. In fact, Minerbe's volume growth constraint becomes a corollary instead of a condition of our first main theorem. In particular, we can now apply his work and improve the curvature decay rate of an ALF instanton to $O\left(r^{-3}\right)$. Therefore, the asymptotic rate can be improved to any $\delta<1$. For ALH non-splitting instantons, we can also improve the curvature decay rate. It turns out that the metric must converge to the flat model exponentially. For more details, see Theorem 4.18. We believe there is a similar self improvement for ALG instantons, but we will leave it for future study.

For the second question, the crucial point is to understand "the end" holomorphically. In the International Congress of Mathematicians 1978, Yau conjectured that every complete Calabi-Yau manifold can be compactified in the complex analytic sense [39]. There are counterexamples if we only assume the completeness without a fast curvature decaying condition [1]. However, when we assume the faster than quadratic curvature decay condition, in both the ALG and ALH non-splitting cases, we can prove Yau's conjecture. In the higher dimension $n \geqslant 3$, assuming the curvature exponentially decays and the metric is asymptotically cylindrical, Haskins, Hein, and Nordström [19] constructed a compactification and therefore verified Yau's conjecture in their settings.

Theorem 1.2. (Main Theorem 2) For any ALG or ALH non-splitting gravitational instanton $M$, there exists a compact elliptic surface $\bar{M}$ with a meromorphic function $u: \bar{M} \rightarrow \mathbb{C P}^{1}$ whose generic fiber is torus. The fiber $D=\{u=\infty\}$ is regular if $M$ is ALH, whereas it is either regular or of type $\mathrm{I}_{0}^{*}$, II, II*, III, III*, IV, IV* if $M$ is ALG. There exists an $\left(a_{1}, a_{2}, a_{3}\right)$ in $\mathbb{S}^{2}$ such that when we use $a_{1} I+a_{2} J+a_{3} K$ as the complex structure, $M$ is biholomorphic to $\bar{M} \backslash D$.

The converse problem is actually very well known and has been studied actively: Given a compact complex manifold $\bar{M}$ and $D$ an anti-canonical divisor, do we have a complete Ricci-flat Kähler metric on $\bar{M} \backslash D$ ? Tian-Yau [38] proved that, for a quasiprojective surface $M=\bar{M} \backslash D$ with $\bar{M}$ smooth and $D$ a smooth anticanonical divisor in $\bar{M}$, as long as $D^{2} \geqslant 0, M$ has a complete Ricci-flat Kähler metric, which has the volume growth of linear order (i.e. ALH). In [22], Hein generalized Tian-Yau's work and constructed ALG gravitational instantons on the complement of the anticanonical 
divisor to the rational elliptic surface $\bar{M} \backslash D$. We do not know whether one can repeat the Hein-Tian-Yau construction for a general elliptic surface, which is not algebraic. The complete understanding of ALG and ALH non-splitting gravitational instantons is even more difficult.

In addition, using gauge theory, Biquard-Boalch [4] also constructed a complete hyperkähler 4-manifold. It is also closely related to the complement of the anticanonical divisor to the rational elliptic surface through the Painlevé equations [6], [37], but it is still unclear whether the Biquard-Boalch metric is ALG or not.

In the ALF case, more discussions are needed:

(1) In the ALF- $A_{k}$ case, Minerbe [35] proved that any ALF- $A_{k}$ instanton must be the trivial product or the multi-Taub-NUT metric. In particular, there is no ALF- $A_{k}$ instantons for $k<-1$.

(2) In the ALF- $D_{k}$ case, Biquard and Minerbe [5] proved that there is no ALF- $D_{k}$ instantons for $k<0$. For $k \geqslant 0$, the first example was constructed by Atiyah and Hitchin [2], where $k=0$. Ivanov and Roček [25] conjectured a formula for larger $k$ using the generalized Legendre transform developed by Lindström and Roček. This conjecture was proved by Cherkis and Kapustin [12], and computed more explicitly by Cherkis and Hitchin [11]. It is conjectured that any ALF- $D_{k}$ instanton must be exactly the metric constructed by them. This conjecture has not been solved yet. However, we are able to prove the existence of the $\mathcal{O}(4)$ multiplet, which plays an important role in the Cherkis-Hitchin-Kapustin-Ivanov-Lindström-Roček construction.

TheOREM 1.3. (Main Theorem 3) In the ALF- $D_{k}$ case, there exists a holomorphic map from the twistor space of $M$ to the total space of the $\mathcal{O}(4)$ bundle over $\mathbb{C P}^{1}$, which commutes with both the projection to $\mathbb{C P}^{1}$ and the real structure.

For the definitions of the twistor space and the real structure, see Theorems 4.24 and 4.25 .

One of our main tools comes from the equivalence between the hyperkähler condition and the Calabi-Yau condition. Actually, for hyperkähler manifolds, we have three complex structures: $I, J$, and $K$. They induce three symplectic forms by

$$
\omega_{1}(X, Y)=g(I X, Y), \quad \omega_{2}(X, Y)=g(J X, Y), \quad \text { and } \quad \omega_{3}(X, Y)=g(K X, Y) .
$$

The form $\omega^{+}=\omega_{2}+i \omega_{3}$ is a $I$-holomorphic symplectic form. This induces the equivalence of $\operatorname{Sp}(1)$ and $\mathrm{SU}(2)$. Notice that, for any $\left(a_{1}, a_{2}, a_{3}\right) \in \mathbb{S}^{2}, a_{1} I+a_{2} J+a_{3} K$ is a Kähler structure. There is a special property of $\operatorname{Sp}(1)$ : Given any vectors $v, w \in T_{p}$, which are orthogonal to each other and have the same length, there exists an $\left(a_{1}, a_{2}, a_{3}\right)$ in $\mathbb{S}^{2}$ such that $\left(a_{1} I+a_{2} J+a_{3} K\right) v=w$. We will use this property to find the best complex structure. 
We obviously benefited from studying a series of papers by Minerbe [33], [34], and [35]. Although his work seems only valid in the ALF- $A_{k}$ case, we manage to make some modest progress in all cases in the present work.

\section{Acknowledgement}

Both authors are grateful for the insightful and helpful discussions with Sir Simon Donaldson, Blaine Lawson, Claude LeBrun, and Martin Roček. We also thank Gilles Carron and $\mathrm{Yu} \mathrm{Li}$ for their suggestions of improvements in the first version of this paper.

The first author was offered full support as a teaching assistant and a full tuition scholarship when he was a graduate student at Stony Brook University. The second author was partially supported by the National Science Foundation through grant no. 1211652.

\section{Notation and definitions}

First, let us understand the standard models near infinity. The explicit expression of those models is defined in Theorem 3.19. To avoid singularity, a ball $B_{R}$ is always removed.

Example. Let $\left(X, h_{1}\right)$ be any manifold of dimension $3-k$ with constant sectional curvature 1 and let $C(X)$ be its metric cone with the standard flat metric $d r^{2}+r^{2} h_{1}$. Let $\mathbb{T}^{k}$ be a $k$-dimensional flat torus. Then, the $\mathbb{T}^{k}$ fibration $E$ over $C(X) \backslash B_{R}$ with a $\mathbb{T}^{k}$ invariant metric $h$ provides the standard model near infinity.

(1) $C(X)=\mathbb{R}^{4} / \Gamma$, where $\Gamma$ is a discrete subgroup in $\mathrm{SU}(2)$ acting freely on $\mathbb{S}^{3}$. In this case, $(E, h)=C(X) \backslash B_{R}$ with the flat metric. It is called ALE.

(2) $C(X)=\mathbb{R}^{3}$, and $(E, h)$ is either the trivial product $\left(\mathbb{R}^{3} \backslash B_{R}\right) \times \mathbb{S}^{1}$ or the quotient of the Taub-NUT metric with mass $m$ outside a ball by $\mathbb{Z}_{|e|}$, where $m e<0$. It is called $\operatorname{ALF}-A_{k}$, with $k=-1$ in the first case and $k=-e-1$ in the second case.

(3) $C(X)=\mathbb{R}^{3} / \mathbb{Z}_{2}$, and $(E, h)$ is either the $\mathbb{Z}_{2}$ quotient of the trivial product of $\mathbb{R}^{3} \backslash B_{R}$ and $\mathbb{S}^{1}$ or the quotient of the Taub-NUT metric with mass $m$ outside a ball by the binary dihedral group $D_{4|e|}$ of order $4|e|$, where $m e<0$. It is called ALF- $D_{k}$ with $k=2$ for the first case, and $k=-e+2$ for the second case.

(4) $C(X)$ is the flat cone $\mathbb{C}_{\beta}$ with a cone angle $2 \pi \beta$, and $(E, h)$ is a torus bundle over $\mathbb{C}_{\beta} \backslash B_{R}$ with a flat metric, where $(\beta, E, h)$ is in the list of some special values. It is called ALG.

(5) $C(X)=\mathbb{R}$, and $(E, h)$ is the product of $\mathbb{R} \backslash B_{R}$ and a flat 3 -torus. It is called ALH splitting. 
(6) $C(X)=\mathbb{R}_{+}$, and $(E, h)$ is the product of $[R,+\infty)$ and a flat 3 -torus. It is called ALH non-splitting.

We may call such fiberation a standard model near infinity. It serves as an asymptotic model in the following sense.

Definition 2.1. A complete Riemannian manifold $(M, g)$ is called asymptotic to the standard model $(E, h)$ of order $\delta$ if there exist a bounded domain $K \subset M$, and a diffeomorphism $\Phi: E \rightarrow M \backslash K$ such that

$$
\Phi^{*} g=h+O^{\prime}\left(r^{-\delta}\right)
$$

for some $\delta>0$.

Any manifold asymptotic to the standard ALE model is called ALE. It stands for asymptotically locally Euclidean. Similarly, any manifold asymptotic to the standard ALF model is called ALF. It means asymptotically locally flat. The ALG and ALH manifold are defined similarly. The letters "G" and " $\mathrm{H}$ " do not have any meanings. They are just the letters after "E" and "F".

Notice that our definition of ALH manifold is different from Hein's definition in [22]. However, Theorem 4.18 implies that there is no essential difference for gravitational instantons.

Notation. We have that $o$ is a fixed point in $M$. In $\S 3, r(p)=\operatorname{dist}(o, p)$ is the geodesic distance between $o$ and $p$. In $\S 4, E$ is a fiberation over

$$
C(X) \backslash B_{R}=\{(r, \theta): r \geqslant R, \theta \in X\} .
$$

So, the pull-back of $r$ by the projection is a function on $E$. On $M$, we pull back that function, cut it off by some smooth function, and add 1 to get the smooth function $r \geqslant 1$. The reader should be careful about the changing meanings of $r$ in various sections of our paper.

$O^{\prime}\left(r^{\alpha}\right)$ means that, for any $m \geqslant 0$, the $m$ th derivative of the tensor is $O\left(r^{\alpha-m}\right)$. Then, $\chi$ will be a smooth cut-off function from $(-\infty,+\infty)$ to $[0,1]$ such that $\chi \equiv 1$ on $(-\infty, 1]$ and $\chi \equiv 0$ on $[2, \infty)$. We will always use $\Delta=-\nabla^{*} \nabla$ as the Laplacian operator.

\section{Asymptotic fibration}

In this section, we prove Main Theorem 1. It is essentially a theorem in Riemannian geometry. The basic tool is to view a ball in the manifold $M$ as a quotient of the ball inside the tangent space, equipped with the metric pulled back from exponential map, by 
the group of local covering transforms corresponding to the short geodesic loops in $M$. In the first subsection, we discuss this picture. In the second subsection, we provide a rough estimate of the holonomy of short geodesic loops. In the third subsection, we use that rough estimate to classify the tangent cone at infinity. In the fourth subsection, we use this information to get a better control of the geodesic loops. Finally, we use this better control to prove our Main Theorem 1.

\subsection{Short geodesic loops and the local covering space}

In 1978, Gromov [18] started the research of almost flat manifolds (i.e., manifolds with very small curvature). In 1981, Buser and Karcher wrote a book [7] to explain Gromov's ideas in detail. In 1982, Ruh [36] provided a new way to understand it. Let $p$ be a point in $M$. The exponential map exp: $T_{p} \rightarrow M$ is a local covering map inside the conjugate radius. We can pull back the metric from $M$ using the exponential map inside the conjugate radius. There is a lemma about the local geometry on the tangent space.

LEMMA 3.1. Let $g_{i j}$ be a metric on $B_{1}(0) \subset \mathbb{R}^{n}$ satisfying the following conditions:

(1) The curvature is bounded by $\Lambda^{2}$;

(2) $g_{i j}(0)=\delta_{i j}$;

(3) The line $\gamma(t)=t \mathbf{u}$ is always a geodesic for any unit vector $\mathbf{u}$.

Then, there exist constants $\Lambda(n)$ and $C(m, n)$ such that, as long as $\Lambda \leqslant \Lambda(n)<\frac{1}{2} \pi$,

(1) Any two points $x$ and $y$ in $B_{1}(0)$ can be connected by a unique minimal geodesic inside $B_{1}(0)$;

(2) If the Ricci curvature is identically zero, then

$$
\left|D^{m}\left(g_{i j}(x)-\delta_{i j}\right)\right|<C(m, n) \Lambda^{2}
$$

for all $m \geqslant 0$ and $x \in B_{1 / 2}$.

Proof. (1) It was proved by Buser and Karcher as [7, Proposition 6.4.6].

(2) Therefore, all the works in [26] apply. We can find functions $l_{i}$ satisfying

$$
\left|\nabla l_{i}(x)-e_{i}(x)\right| \leqslant C(n) \Lambda^{2} \quad \text { and } \quad\left|\nabla^{2} l_{i}(x)\right| \leqslant C(n) \Lambda^{2}
$$

for all $x \in B_{1 / 2}(0)$ as long as $\Lambda(n)$ is small enough, where $e_{i}(x)$ is a vector field that is parallel along radical geodesics and equals to $\partial / \partial x_{i}$ at origin. For even smaller $\Lambda(n)$, we can use $l_{i}$ as coordinate functions in

$$
L_{0.9}(0)=\left\{\sum_{i} l_{i}^{2}<(0.9)^{2}\right\} \subset B_{1}(0)=\left\{\sum_{i} x_{i}^{2}<1\right\} .
$$


In this coordinate,

$$
\begin{aligned}
\left.\left|g_{i j} w^{i} w^{j}-\right| w\right|^{2} \mid & \leqslant C(n) \Lambda^{2}|w|^{2} \leqslant .01|w|^{2}, \\
\left|\partial_{k} g_{i j}\right| & <C(n) \Lambda^{2}<1 \\
\Delta u & =\frac{1}{\sqrt{G}} \partial_{j}\left(\sqrt{G} g^{i j} \frac{\partial u}{\partial l_{i}}\right) .
\end{aligned}
$$

What is more, $\left|\Delta l_{i}\right|<C(n) \Lambda^{2}$. By [16, Theorem 9.15], for all $1<p<\infty$, there is a unique solution $u_{i} \in W^{2, p}\left(L_{0.9}\right) \cap W_{0}^{1, p}\left(L_{0.9}\right)$ such that $\Delta u_{i}=\Delta l_{i}$. By [16, Lemma 9.17], we actually have

$$
\left\|u_{i}\right\|_{W^{2, p}\left(L_{0.9}(0)\right)}<C(n, p)\left\|\Delta l_{i}\right\|_{L^{p}\left(L_{0.9}(0)\right)}<C(n, p) \Lambda^{2} .
$$

By the Sobelev embedding theorem (cf. [16, Theorem 7.26]),

$$
\left\|u_{i}\right\|_{C^{1}\left(\overline{\left.L_{0.9}(0)\right)}\right.}<C(n)\left\|u_{i}\right\|_{W^{2,2 n}\left(L_{0.9}(0)\right)}<C(n) \Lambda^{2} .
$$

In particular, when $\Lambda(n)$ is small enough, $h_{i}=l_{i}-u_{i}$ gives a harmonic coordinate in

$$
H_{0.8}(0):=\left\{\sum_{i} h_{i}^{2}<(0.8)^{2}\right\} \subset L_{0.9}(0) .
$$

In this harmonic coordinate,

$$
\frac{1}{1.02}|w|^{2}<g_{i j} w^{i} w^{j}<1.02|w|^{2} .
$$

By elliptic regularity, all of the above functions are actually smooth. So, we can differentiate them to get equations. Since $\Gamma_{i j}^{k} g^{i j}=0$, we know that

$$
2 \mathrm{Ric}_{m k}=g^{i m} R_{i j k l} g^{j l}+g^{i k} R_{i j m l} g^{j l}
$$

satisfies

$$
g^{r s} \frac{\partial^{2}\left(g_{i j}-\delta_{i j}\right)}{\partial h_{r} \partial h_{s}}=-2 \operatorname{Ric}_{i j}+Q_{i j}(g, \partial g)+Q_{j i}(g, \partial g),
$$

where

$$
Q_{m k}(g, \partial g)=g^{j l} \partial_{l} g_{i m} \Gamma_{k j}^{i}-g^{j l} g_{i m} \Gamma_{k j}^{h} \Gamma_{l h}^{i}-g_{i m} \partial_{k} g^{j l} \Gamma_{j l}^{i} .
$$

We already know that

$$
\left\|g_{i j}-\delta_{i j}\right\|_{W^{1, p}\left(H_{0.8}(0)\right)}<C(n) \Lambda^{2}
$$

from the $W^{2, p}$ bound of $u_{i}$. So,

$$
\left\|Q_{i j}(g, \partial g)\right\|_{L^{p / 2}\left(H_{0.8}(0)\right)}<C(n) \Lambda^{4} .
$$


When the Ricci curvature is identically zero, by [16, Theorem 9.11], we have

$$
\left\|g_{i j}-\delta_{i j}\right\|_{W^{2, p / 2}\left(H_{0.7}\right)}<C(n)\left(\left\|g_{i j}-\delta_{i j}\right\|_{L^{p / 2}\left(H_{0.8}\right)}+\left\|Q_{i j}\right\|_{L^{p / 2}\left(H_{0.8}\right)}\right)<C(n) \Lambda^{2} .
$$

After taking more derivatives, we can get the required bound in the harmonic coordinate. This in turn bounds the Christoffel symbol and gives a bound of the geodesic equation. So, when we solve this geodesic equation, we can get the required bound in the geodesic ball.

The above estimate is an interior estimate. The number $\frac{1}{2}$ can be replaced by any number smaller than 1.

To find out the local covering transform, we look at the preimage $p_{1}$ of $p$ under the exponential map inside $B_{1}(0)$. There is a local covering transform $F$ that maps zero to $p_{1}$. The image of the radical geodesic from zero to $p_{1}$ is a geodesic loop based at $p$. This gives a one-to-one correspondence between short geodesic loops and covering transforms.

Now suppose that we have two short-enough geodesic loops $\gamma_{1}$ and $\gamma_{2}$ with the same base point $p$. Then, they correspond to two local covering transforms $F_{1}$ and $F_{2}$. The composition $F_{1} \circ F_{2}$ is also a local covering transform. It corresponds to another geodesic loop based at $p$. It is exactly the product of $\gamma_{1}$ and $\gamma_{2}$ defined by Gromov.

For any $q$ close enough to $p$, choose a preimage $q_{0}$ of $q$ close enough to zero. Then, $q_{1}=F\left(q_{0}\right)$ is another preimage of $q$, which is very close to $p_{1}$. The image of the shortest geodesic connecting $q_{1}$ and $q_{2}$ under the exponential map is a geodesic loop based at $q$. It is called the sliding of $\gamma$. When $q$ moves along a curve $\alpha$, the sliding of $\gamma$ becomes a 1-parameter family of curves. It is called the sliding of $\gamma$ along the curve $\alpha$.

When we parallel transport any vector $v$ along the geodesic loop, we will get another vector $\operatorname{hol}(v)$. The function hol: $T_{p} \rightarrow T_{p}$ is called the holonomy of the loop. For hyperkähler manifolds, hol $\in \mathrm{Sp}(1)=\mathrm{SU}(2)$. Under a suitable orthonormal basis, any element in $\mathrm{SU}(2)$ can be written as

$$
\mathbf{A}=\left(\begin{array}{cc}
e^{i \theta} & 0 \\
0 & e^{-i \theta}
\end{array}\right)
$$

So,

$$
\mathbf{A}-\mathbf{I d}=\left(\begin{array}{cc}
e^{i \theta}-1 & 0 \\
0 & e^{-i \theta}-1
\end{array}\right) \quad \text { and } \quad(\mathbf{A}-\mathbf{I d})\left(\begin{array}{c}
v_{1} \\
v_{2}
\end{array}\right)=\left(\begin{array}{c}
\left(e^{i \theta}-1\right) v_{1} \\
\left(e^{-i \theta}-1\right) v_{2}
\end{array}\right) .
$$

So, $|(\mathbf{A}-\mathbf{I d}) \mathbf{v}|=|\mathbf{A}-\mathbf{I d}||\mathbf{v}|$ if we define the norm by

$$
|\mathbf{A}-\mathbf{I d}|=\left|e^{i \theta}-1\right|=\left|e^{-i \theta}-1\right| .
$$


This property is also a special property of $\mathrm{SU}(2)$. For instance, $\mathrm{SO}(4)$ does not have this property.

In the flat case, local covering transforms are all linear maps. Let $T_{1}(\mathbf{x})=\mathbf{a x}+\mathbf{b}$ and $T_{2}(\mathbf{x})=\mathbf{A x}+\mathbf{B}$ be two local covering transforms, where $\mathbf{a}, \mathbf{A} \in \operatorname{SO}(n)$ and $\mathbf{b}, \mathbf{B} \in \mathbb{R}^{n}$. They correspond to two geodesic loops $\gamma_{1}$ and $\gamma_{2}$ with the same base point $p$. Note that $\mathbf{A}$ and $\mathbf{a}$ are exactly the holonomy of $\gamma_{1}$ and $\gamma_{2}$, whereas $|\mathbf{B}|$ and $|\mathbf{b}|$ are the same as the length of loops $\gamma_{1}$ and $\gamma_{2}$, respectively. We have that

$$
T_{1} \circ T_{2}(\mathbf{x})=\mathbf{a}(\mathbf{A} \mathbf{x}+\mathbf{B})+\mathbf{b}=\mathbf{a A} \mathbf{x}+\mathbf{a B}+\mathbf{b}
$$

will correspond to the Gromov product of $\gamma_{1}$ and $\gamma_{2}$. So,

$$
T_{1}^{-1} T_{2}^{-1} T_{1} T_{2}(\mathbf{x})=\mathbf{a}^{-1} \mathbf{A}^{-1} \mathbf{a A x}+\mathbf{a}^{-1} \mathbf{A}^{-1}((\mathbf{a}-\mathbf{I d}) \mathbf{B}+(\mathbf{I d}-\mathbf{A}) \mathbf{b}) .
$$

The Lie algebra also consists of linear maps. Taking the derivative in the above expression of the commutator at the origin

$$
T_{1}(\mathbf{x})=T_{2}(\mathbf{x})=\operatorname{Id}(\mathbf{x})=\operatorname{Id}(\mathbf{x})+\mathbf{0},
$$

the Lie bracket is

$$
[\mathbf{a x}+\mathbf{b}, \mathbf{A x}+\mathbf{B}]=[\mathbf{a}, \mathbf{A}] \mathbf{x}+(\mathbf{a B}-\mathbf{A b}) .
$$

In a general case, we can understand the covering transform in the following way: We start from $q_{0}$ in $B_{1}(0) \subset T_{p}(M)$. Then, the exponential map at $p$ maps the point $p_{1} \in B_{1}(0)$ to $p \in M$. The derivative maps the tangent vector at $p_{1}$ to the tangent vector at $p$. Let $\tilde{A}$ be the inverse of the map. Then, $F\left(q_{0}\right)=\exp _{p_{1}}\left(\tilde{A} q_{0}\right)$. In the Ricci flat case, by Lemma 3.1, $g_{i j}$ and its $m$ th derivatives are bounded by $C(m, n) \Lambda^{2}$. So, the Christoffel symbols and their higher derivatives are also bounded. By the property of ODE, all of the parallel transports, the geodesic equations, and their higher derivatives have the same kind of bound. In particular, the difference between $\tilde{A}$ and the holonomy $A$ of the geodesic loop is bounded by $C(n) \Lambda^{2}$. The difference between $F\left(q_{0}\right)$ and $p_{1}+\tilde{A} q_{0}$ is bounded by $C(n) \Lambda^{2}$. In conclusion, the difference between $F\left(q_{0}\right)$ and $p_{1}+A q_{0}$ is bounded by $C(n) \Lambda^{2}$, whereas the difference between their higher derivatives is bounded by $C(m, n) \Lambda^{2}$.

From now on, we are back to the gravitational instanton $M$ with the point $o$. We will rescale the ball $B_{\operatorname{dist}(o, p) / 2}(p)$ to a ball with a radius of 1 and apply the theory in this section. In particular, the metric on the local covering space is $\delta_{i j}+O^{\prime}\left(r^{-\epsilon}\right)$. The difference between the local covering transform with the linear map given by the length, direction, and the holonomy of the geodesic loop is $O^{\prime}\left(r^{1-\epsilon}\right)$.

For short loops, there is a better control given by Buser and Karcher as [7, Proposition 2.3.1]. They proved that the rotation (i.e. holonomy) part of Gromov's product of $\gamma_{1}$ and $\gamma_{2}$ is given by the calculation in the flat case with an error bounded by $\mathrm{Cr}^{-2-\epsilon} L\left(\gamma_{1}\right) L\left(\gamma_{2}\right)$, whereas the error of the translation (i.e. length) part is bounded by $C r^{-2-\epsilon} L\left(\gamma_{1}\right) L\left(\gamma_{2}\right)\left(L\left(\gamma_{1}\right)+L\left(\gamma_{2}\right)\right)$. 


\subsection{Control of holonomy of geodesic loops}

In this section, we will use the ODE comparison to study the sliding of geodesic loops and the variation of the induced holonomy. First, let us recall a well-known Jacobi equation,

$$
J^{\prime \prime}(t)=\left(\frac{t}{2}\right)^{-2-\epsilon} J(t)
$$

which satisfies the following property.

Proposition 3.2. (Cf. [17, Theorem C]) Let $J$ be the solution of the Jacobi equation with

$$
J(2)=0 \quad \text { and } \quad J^{\prime}(2)=1 .
$$

Then,

$$
1 \leqslant J^{\prime}(t) \nearrow J^{\prime}(\infty)\left(:=\lim _{t \rightarrow \infty} J^{\prime}(t)\right) \leqslant \exp \int_{2}^{\infty}(t-2)\left(\frac{t}{2}\right)^{-2-\epsilon} d t<\infty
$$

and

$$
t-2 \leqslant J(t) \leqslant J^{\prime}(\infty)(t-2) .
$$

Let $\gamma$ be a geodesic loop based at $p \in M$ and $\alpha$ be an arc-length parameterized curve passing through $p$. Suppose $r=\operatorname{dist}(0, p)=r(p)>3$. As discussed before, we slide $\gamma$ along $\alpha$ and get a 1-parameter family of geodesic loops $\gamma_{t}$ based at $\alpha(t)$. Then, their length and induced holonomy satisfy the following.

Proposition 3.3. Suppose the length and the holonomy of the geodesic loop $\gamma_{t}$ are $L(t)$ and $\operatorname{hol}(t)$, respectively. Then,

$$
\left|L^{\prime}(t)\right| \leqslant|\operatorname{hol}(t)-\mathrm{Id}|
$$

and

$$
|\operatorname{hol}(t)-\mathrm{Id}|^{\prime} \leqslant L(t) \cdot \max _{x \in \gamma_{t}}|\mathrm{Rm}|(x) .
$$

Proof. Let $\gamma(s, t)=\gamma_{t}(s)$, then $\gamma(0, t)=\gamma(1, t)=\alpha(t)$, and for any fixed $t, \gamma(s, t)$ is a geodesic. So,

$$
\partial_{s}:=\gamma_{*}\left(\frac{\partial}{\partial s}\right) \quad \text { and } \quad \partial_{t}:=\gamma_{*}\left(\frac{\partial}{\partial t}\right)
$$

satisfy

$$
\nabla_{\partial_{s}} \partial_{s}=0, \quad\left[\partial_{s}, \partial_{t}\right]=\nabla_{\partial_{s}} \partial_{t}-\nabla_{\partial_{t}} \partial_{s}=0, \quad L(t)=\int_{0}^{1}\left|\partial_{s}\right| d s
$$


Then,

$$
\begin{aligned}
\left.\frac{d L(t)}{d t}\right|_{t=t_{0}} & =\int_{0}^{1} \frac{\left\langle\nabla_{\partial_{t}} \partial_{s}, \partial_{s}\right\rangle}{\left\langle\partial_{s}, \partial_{s}\right\rangle^{1 / 2}} d s=\frac{1}{L\left(t_{0}\right)} \int_{0}^{1}\left\langle\nabla_{\partial_{s}} \partial_{t}, \partial_{s}\right\rangle d s \\
& =\frac{1}{L\left(t_{0}\right)} \int_{0}^{1} \nabla_{\partial_{s}}\left\langle\partial_{t}, \partial_{s}\right\rangle d s=\frac{\left.\left\langle\partial_{t}, \partial_{s}\right\rangle\right|_{s=0} ^{s=1}}{L\left(t_{0}\right)}=\left\langle\alpha^{\prime}\left(t_{0}\right), \frac{(\text { hol }- \text { Id })\left[\partial_{s}\left(0, t_{0}\right)\right]}{L\left(t_{0}\right)}\right\rangle .
\end{aligned}
$$

So,

$$
\left|L^{\prime}\right| \leqslant \mid \text { hol }-\mathrm{Id} \mid
$$

Moreover, given any unit length vector $V$ at $\gamma\left(0, t_{0}\right)$, we can parallel transport it along $\alpha(t)=\gamma(0, t)$, and then parallel transport it along $\gamma_{t}$. Then, $\operatorname{hol}(V(0, t))=V(1, t)$. So,

$$
|| \text { hol }-\left.\mathrm{Id}\right|^{\prime}|\leqslant| \nabla_{\partial_{t}} V(1, t)\left|\leqslant \int_{0}^{1}\right| \nabla_{\partial_{s}} \nabla_{\partial_{t}} V\left|=\int_{0}^{1}\right| R\left(\partial_{s}, \partial_{t}\right) V(s, t)\left|\leqslant \max _{x \in \gamma_{t}}\right| \operatorname{Rm} \mid L .
$$

TheOREM 3.4. For any geodesic loop based at $p$ with $r=r(p)=d(p, o)>3$ and length $L \leqslant C_{1} r$, the holonomy along the loop satisfies

$$
\mid \text { hol }-\mathrm{Id} \mid \leqslant \frac{J^{\prime}(r)}{J(r)} L \leqslant C_{2} \frac{L}{r}
$$

where the constants are

$$
C_{1}=\frac{1}{2} \inf _{t>2} \frac{t}{J(t)} \inf _{t>3} \frac{J(t)}{t} \quad \text { and } \quad C_{2}=\sup _{t>3} J^{\prime}(t) \sup _{t>3} \frac{t}{J(t)} .
$$

Proof. If we choose $\alpha(t)$ so that

$$
\partial_{t}=\frac{\text { hol }- \text { Id }}{\mid \text { hol }- \text { Id } \mid} \frac{\partial_{s}}{L\left(t_{0}\right)},
$$

we can get $L^{\prime}(t)=\mid$ hol $-\mathrm{Id} \mid$. It is some kind of gradient flow. The other fundamental equation is that $|\mathrm{hol}-\mathrm{Id}|^{\prime}$ is bounded by the product of $L$ and the maximal Riemannian curvature along the geodesic loop.

Given $p$ whose distance to origin $r=r(p)=d(p, o)>3$ and any geodesic loop based at $p$ with a length smaller than $C_{1} r<\frac{1}{2} r$, if

$$
\mid \text { hol }-\mathrm{Id} \mid>\frac{J^{\prime}(r)}{J(r)} L,
$$

we can slide the curve back along the gradient flow. In other words, we start from $\alpha(r)=p$ and get the curve $\alpha:\left[t_{1}, r\right] \rightarrow M$, as well as the corresponding $\gamma_{t}$. Let $t_{1}$ be the biggest $t_{1}$ such that one of the following happens:

(1) $L\left(t_{1}\right)=\frac{1}{2} t_{1}$;

(2) $L^{\prime}\left(t_{1}\right)=\mid$ hol $-\mathrm{Id} \mid=0$ or $L\left(t_{1}\right)=0$;

(3) $t_{1}=2$. 
Then, when $t \in\left(t_{1}, r\right)$, we have $0<L(t)<\frac{1}{2} t$ and $t>t_{1} \geqslant 2$. So, the distance to the origin is at least $t-L(t)>\frac{1}{2} t$, the curvature is bounded by $\left(\frac{1}{2} t\right)^{-2-\epsilon}$, and the conjugate radius is at least $\pi\left(\frac{1}{2} t\right)^{1+\epsilon / 2}>\frac{1}{2} t>L(t)$. So, the geodesic loop can exist without going out of the conjugate radius. Combining two fundamental equations together, we have

$$
L^{\prime \prime}(t) \leqslant L(t) \max |\operatorname{Rm}| \leqslant L(t)(t-L(t))^{-2-\epsilon}<L(t)\left(\frac{1}{2} t\right)^{-2-\epsilon}, \quad \text { for all } t \in\left(t_{1}, r\right) .
$$

Therefore, $\left(L^{\prime} J-J^{\prime} L\right)^{\prime}=L^{\prime \prime} J-J^{\prime \prime} L<0$. By our hypothesis,

$$
L^{\prime}(r)>\frac{J^{\prime}(r)}{J(r)} L(r)
$$

So,

$$
L^{\prime}(t) J(t)-J^{\prime}(t) L(t)>0 \Longrightarrow\left(\frac{L(t)}{J(t)}\right)^{\prime}>0 \Longrightarrow \frac{L(t)}{J(t)}<\frac{L(r)}{J(r)}, \quad \text { for all } t \in\left[t_{1}, r\right) .
$$

Thus,

$$
L\left(t_{1}\right)<\frac{L(r)}{J(r)} J\left(t_{1}\right) \leqslant C_{1} \frac{r}{J(r)} \frac{J\left(t_{1}\right)}{t_{1}} t_{1} \leqslant \frac{t_{1}}{2}
$$

and $L^{\prime}\left(t_{1}\right) J\left(t_{1}\right)>J^{\prime}\left(t_{1}\right) L\left(t_{1}\right) \geqslant 0$. In other words, $t_{1}=2$. But then

$$
L(2)<\frac{L(r)}{J(r)} J(2)=0 .
$$

It is a contradiction.

For any fixed geodesic ray $\alpha$ starting from $o$, any number $r>3$, and any geodesic loop $\gamma$ based at $p=\alpha(r)$ with length $L \leqslant C_{1} r$, when we slide it along the ray toward infinity, it will always exist (i.e., stay within the conjugate radius). This follows from the following rough estimate.

COROLLARY 3.5. The length $L(t)$ of the geodesic loop based at $\alpha(t)$ is smaller than $\frac{1}{2} t$ for all $t \geqslant r$.

Proof. By Proposition 3.3 and Theorem 3.4, we know that

$$
L^{\prime}(t) \leqslant \frac{J^{\prime}(t)}{J(t)} L(t)
$$

So,

$$
(\ln L)^{\prime} \leqslant(\ln J)^{\prime} \Longrightarrow L(t) \leqslant \frac{L(r)}{J(r)} J(t) \leqslant \frac{t}{2}, \quad \text { for all } t \geqslant r>3 .
$$

We will derive a better estimate and use it to prove the first main theorem. 


\subsection{Classification of tangent cone at infinity}

To understand how the length of geodesic loops varies, we first need to understand the structure at infinity. Our assumption of the decay of the curvature means that we are at a manifold with asymptotically non-negative curvature. The end of such a manifold is well studied and goes back to Kasue [27]. Here, a complete connected non-compact Riemannian manifold $M$ with a base point $o$ is called asymptotically non-negative curved if there exists a monotone non-increasing function $k:[0, \infty) \rightarrow[0, \infty)$ such that the integral $\int_{0}^{\infty} t k(t) d t$ is finite and the sectional curvature of $M$ at any point $p$ is bounded from below by $-k(\operatorname{dist}(o, p))$. Of course, the gravitational instanton $M$ satisfies this condition.

TheOREM 3.6. ([27], [14], [32]) Let $M$ be a manifold with asymptotically nonnegative curvature. Two rays $\sigma$ and $\gamma$ starting from o are called equivalent if

$$
\lim _{t \rightarrow \infty} \frac{\operatorname{dist}(\sigma(t), \gamma(t))}{t}=0
$$

Denote the set of equivalent classes of geodesic rays starting from o by $S(\infty)$. Then, there exists a metric $\delta_{\infty}$ on $S(\infty)$ such that $\left(S(\infty), \delta_{\infty}\right)$ forms a compact inner metric space, in other words, a length space. Consider the cone $C(S(\infty))$ over $S(\infty)$ with the natural distance

$$
\Delta_{\infty}\left((t, p),\left(t^{\prime}, p^{\prime}\right)\right)=\sqrt{t^{2}+t^{\prime 2}-2 t t^{\prime} \cos \left(\min \left\{\pi, \delta_{\infty}\left(p, p^{\prime}\right)\right\}\right)} .
$$

Fix the representative $\sigma$ from each equivalent class $[\sigma]$. Define the map

$$
\Phi_{t}:\{r \in[a, b]\} \cap C(S(\infty)) \longrightarrow\{r \in[a t, b t]\} \cap M
$$

by $\Phi_{t}(r,[\sigma])=\sigma(r t)$ for any fixed $0<a<b<\infty$ and any $t>0$. Then, the Gromov-Hausdorff distance between $\left(\{r \in[a, b]\} \cap C(S(\infty)), \Delta_{\infty}\right)$ and $(\{r \in[a t, b t]\} \cap M$, dist $/ t)$ using the map $\Phi_{t}$ converges to zero when $t$ goes to infinity. In other words, the tangent cone at infinity is unique and must be a metric cone $C(S(\infty))$.

Remark. Drees [14] pointed out a gap in [27]. It was corrected by Mashiko, Nagano, and Otsuka [32].

The following additional thing is true for gravitational instantons.

THEOREM 3.7. (ALH splitting) If the $S(\infty)$ of a gravitational instanton $M$ has more than one connected component, $M$ must be isometric to the product of $\mathbb{R}$ and a flat 3-torus. 
Proof. If $S(\infty)$ has more than one connected component, we can find a large-enough ball $B_{R}$ and two sequences $p_{i}$ and $q_{i}$ such that $\operatorname{dist}\left(o, p_{i}\right) \rightarrow \infty$, dist $\left(o, q_{i}\right) \rightarrow \infty$, and any minimal geodesics connecting $p_{i}$ and $q_{i}$ must pass through $B_{R}$ for any $i$ large enough. By compactness of $B_{R}$, the minimal geodesics converge to a line. Notice that $M$ is Ricci-flat, so the splitting theorem [10] implies that $M$ must be isometric to the product of $\mathbb{R}$ and a 3-manifold. The 3-manifold is also Ricci-flat and therefore flat. Now, any geodesic loop in this 3-manifold must have the trivial holonomy by Theorem 3.4. So, it must be a 3 -torus.

From now on, we assume that $S(\infty)$ has only one component.

As a corollary, the following is true.

Corollary 3.8. Fix a ray $\gamma$ starting from o. There is a constant $C_{3}$ such that, for any point $p$ in the large-enough sphere $S_{r(p)}$, there is a curve within $B_{1.1 r(p)} \backslash B_{0.9 r(p)}$ connecting $p$ and $\gamma(r(p))$ with a length bounded by $C_{3} r(p)$.

There is more information about the tangent cone at infinity of the gravitational instanton $M$.

THEOREM 3.9. The tangent cone at infinity $C(S(\infty))$ of the gravitational instanton $M$ must be a flat manifold with only possible singularity at the origin.

Proof. Pick $p \in C(S(\infty)) \backslash\{o\}$. We may find $p_{i} \in M$ such that $p_{i} \rightarrow p$ in the GromovHausdorff sense. Pick some small-enough number $\kappa$. For $i$ large enough, the ball $\left(B_{\kappa r_{i}}\left(p_{i}\right), r_{i}^{-2} g\right)$ is $B_{\kappa} / G_{i}$, where $B_{\kappa}$ is the ball in the Euclidean space with the metric pulled back by the exponential map, and $G_{i}$ is the group of local covering transforms. By Fukaya's result in [15], $G_{i}$ converge to some Lie group $G$, and $B_{\kappa} / G_{i}$ converge to $B_{\kappa} / G$. So, $G$ is a subgroup of $\mathbb{R}^{4} \rtimes \mathrm{SU}(2) \leqslant \operatorname{Iso}\left(\mathbb{R}^{4}\right)$. The action of $G$ on $B_{\kappa}$ corresponds to the action of $G_{i}$ on $B_{\kappa r_{i}}\left(p_{i}\right)$. So, if an element $g \in G \backslash\{\operatorname{Id}\}$ has a fixed point in $B_{\kappa}$, the geodesic loops in $B_{\kappa r_{i}}\left(p_{i}\right)$ corresponding to the sequence $g_{i} \in G_{i}$ converging to $g$ would have large $\mid$ hol - Id $\mid$ compared with their lengths by the relationship between the geodesic loops and covering transforms. This contradicts Theorem 3.4. So, the action of $G$ is free. Therefore, it is enough to look at the Lie algebra $\mathfrak{g}$ (i.e., the infinitesimal part of $G$ ) to determine the local geometry. We have the following cases:

(0) $\operatorname{dim} G=0$. We get $\mathbb{R}^{4}$ locally.

(1) $\operatorname{dim} G=1$. Then, $\mathfrak{g}$ is generated by $\mathbf{x} \mapsto \mathbf{a x}+\mathbf{b}$, where $\mathbf{a} \in \mathfrak{s u}(2)$. Notice that $\mathrm{SU}(2)$ can be identified naturally with the unit sphere of quaternions. Then, $\mathfrak{s u}(2)$ would be the space of pure imaginary quaternions. So, the Lie bracket is exactly twice the cross product in $\mathbb{R}^{3}$.

Note that a must be $\mathbf{O}$ or must be invertible by the property of quaternions. When $\mathbf{a}=\mathbf{O}$, the group $G$ consists of pure translations, and we get $\mathbb{R}^{3}$. 
Otherwise, $\mathbf{a x}+\mathbf{b}=\mathbf{a}\left(\mathbf{x}+\mathbf{a}^{-1} \mathbf{b}\right)$. The fixed point $-\mathbf{a}^{-1} \mathbf{b}$ must be outside of $B_{\kappa}$. The group $G$ is generated by

$$
\mathbf{x} \longmapsto\left(\begin{array}{cc}
e^{i \theta} & 0 \\
0 & e^{-i \theta}
\end{array}\right)\left(\mathbf{x}+\mathbf{a}^{-1} \mathbf{b}\right)-\mathbf{a}^{-1} \mathbf{b} .
$$

If we take the one-to-one correspondence

$$
\mathbf{x} \longmapsto \mathbf{x}+\mathbf{a}^{-1} \mathbf{b}=(x+i y, z+i w) \longmapsto(x+i y, z-i w),
$$

then $G$ becomes

$$
\left(\begin{array}{cc}
e^{i \theta} & 0 \\
0 & e^{i \theta}
\end{array}\right)
$$

So, it is a cone over $\mathbb{S}^{3} / \mathbb{S}^{1}$, where $\mathbb{S}^{3} / \mathbb{S}^{1}$ is the Hopf fiberation. So, $B_{\kappa}$ is a local piece of the cone over $\mathbb{S}^{2}$, in other words, $\mathbb{R}^{3}$, too.

(2) $\operatorname{dim} G=2$. Any 2-dimensional Lie algebra has a basis $e_{1}, e_{2}$ satisfying $\left[e_{1}, e_{2}\right]=c e_{1}$. For $\mathfrak{g}, e_{1}(\mathbf{x})=\mathbf{a x}+\mathbf{b}$ and $e_{2}(\mathbf{x})=\mathbf{A} \mathbf{x}+\mathbf{B}$ must satisfy

$$
[\mathbf{a}, \mathbf{A}] \mathbf{x}+(\mathbf{a B}-\mathbf{A b})=[\mathbf{a x}+\mathbf{b}, \mathbf{A} \mathbf{x}+\mathbf{B}]=c e_{1}=c(\mathbf{a x}+\mathbf{b}) .
$$

Here, $\mathbf{A}, \mathbf{a} \in \mathfrak{s u}(2)$. If $\mathbf{a}=\mathbf{O}$, then $\mathbf{A} \mathbf{b}=-c \mathbf{b}$. So, $\mathbf{A}=\mathbf{O}, G$ consists of pure translations, and we get $\mathbb{R}^{2}$. If $\mathbf{a} \neq \mathbf{O}$, then because $[\mathbf{a}, \mathbf{A}]=c \mathbf{a}$, we must have $\mathbf{a}=\mathbf{A}$ and $c=0$. Therefore, we have $\mathbf{a B}=\mathbf{A} \mathbf{b}=\mathbf{a b}$, and so $\mathbf{B}=\mathbf{b}$, which is a contradiction.

(3) $\operatorname{dim} G=3$. We get $\mathbb{R}^{1}$.

THEOREM 3.10. The tangent cone at infinity $C(S(\infty))$ must be one of the following: (ALE) $\mathbb{R}^{4} / \Gamma$, where $\Gamma$ is a discrete subgroup of $O(4)$ acting freely on $\mathbb{S}^{3}$;

$\left(\mathrm{ALF}-A_{k}\right) \mathbb{R}^{3}$

(ALF- $\left.D_{k}\right) \mathbb{R}^{3} / \mathbb{Z}_{2}$, that is a cone over $\mathbb{R} \mathbb{P}^{2}$;

(ALG) flat cone with angle $\in(0,2 \pi]$;

(ALH non-splitting) $\mathbb{R}_{+}$.

Proof. By Theorem 3.6, the tangent cone at infinity is unique and must be a metric cone $C(S(\infty))$. By Theorems 3.7 and $3.9, S(\infty)$ is a connected manifold because we have assumed that $M$ is not ALH splitting.

(ALH non-splitting) If $S(\infty)$ is zero-dimensional, $C(S(\infty))$ must be $\mathbb{R}_{+}$.

(ALG) If $S(\infty)$ is 1-dimensional, $C(S(\infty))$ is a flat cone. If the cone angle is bigger than $2 \pi$, it contains a line, so there is a contradiction from the almost splitting theorem (cf. [8, Theorem 6.64]).

(ALF) If $S(\infty)$ is 2-dimensional, $S(\infty)$ must be a 2-manifold with constant positive curvature 1 . So, its universal cover is the space form $\mathbb{S}^{2}$. Thus, $S(\infty)=\mathbb{S}^{2} / \Gamma$, where the 
group of covering transforms $\Gamma$ is a subgroup of $\operatorname{Iso}\left(\mathbb{S}^{2}\right)=\mathrm{O}(3)$ acting freely. Now, pick any element $A$ in $\Gamma, A^{2} \in \mathrm{SO}(3)$. However, any element in $\mathrm{SO}(3)$ has a fixed point, so $A^{2}=\mathrm{Id}$. Thus, $A= \pm \mathrm{Id}$. Therefore, $S(\infty)=\mathbb{S}^{2}$ (the $A_{k}$ case) or $\mathbb{R} \mathbb{P}^{2}$ (the $D_{k}$ case).

(ALE) If $S(\infty)$ is 3-dimensional, $S(\infty)$ must have constant sectional curvature, too. Its universal cover is the space form, too. So, $C(S(\infty))=\mathbb{R}^{4} / \Gamma$, where $\Gamma$ is a discrete subgroup of $O(4)$ acting freely on $\mathbb{S}^{3}$.

From now on, we use the terminology of ALE, ALF, ALG, and ALH to distinguish the different type of the (unique) tangent cone at infinity. Those terminologies make sense after we prove more properties.

THEOREM 3.11. In the ALE case, $M$ has the maximal volume growth rate, and it is in Kronheimer's list.

Proof. By Colding's volume convergence theorem [13], $M^{4}$ has the maximal volume growth rate. Moreover, the faster than quadratic curvature decay condition ensures that $\int_{M}|\mathrm{Rm}|^{2}<\infty$. So, by Bando-Kasue-Nakajima's work [3], $M$ is ALE of order 4. Thus, Kronheimer's works in [29] and [30] apply.

\subsection{Decomposing geodesic loops into basis}

Before proceeding, we need a theorem about Lie groups. For any Lie group $H$, the exponential map exp from a small ball $B_{\kappa}=B_{\kappa}(o)$ in its Lie algebra $\mathfrak{h}$ to $H$ is a bijection. We call the inverse of exp to be log. If there is no ambiguity, the length of $g \in H$ will mean $|\log g|$.

Theorem 3.12. ([7, Theorem 4.5]) Suppose that $H$ is a Lie group, $G_{i}$ are discrete subgroups of $H$ converging to a $k$-dimensional Lie subgroup $G$ of $H$. Then, for $i$ large enough and $\kappa$ small enough, there exist $k$ elements $g_{i, j}(j=1,2, \ldots, k)$ such that $\left|\log g_{i, j}\right|$ converge to zero as $i$ goes to infinity, and all elements in $B_{\kappa}(\mathrm{Id}) \cap G_{i}$ are generated by $g_{i, j}$. What is more, for any fixed large-enough $i$, the angles between $\log g_{i, j}$ are bounded from below by a small positive number independent of $i$. In addition, the commutator of $g_{i, a}$ and $g_{i, b}$ is generated by $g_{i, c}$ for $c=1,2, \ldots, \min \{a, b\}-1$. In particular, $g_{i, 1}$ commutes with others.

Remark. According to [7, Theorem 4.5], it is enough to assume that $G_{i}$ have a local group structure near the identity rather than being a group. Actually, the theorem is true even if the product of $a, b \in G_{i}$ contains an error controlled by $C_{i}|a||b|$, where $C_{i}$ converge to zero as $i$ goes to infinity. In particular, the local groups $G_{i}$ in Theorem 3.9 satisfy the theorem. For those local groups, since the rotation (i.e., holonomy) part is 
bounded by the translation (i.e., length) part by Theorem 3.4, the length of the geodesic loop is equivalent to the length in the above theorem.

Now, we are ready to go back to study the length of short geodesic loops. In the rest of this section, we fix a geodesic ray $\alpha$ from $o$ to infinity and begin doing the analysis of geodesic loops based on the ray. This ray corresponds to the point $(1,[\alpha])$ in the tangent cone at infinity $C(S(\infty))$.

TheOREM 3.13. In the ALF- $A_{k}$ or ALF- $D_{k}$ cases, there is a geodesic loop $\gamma_{1}$ such that when we slide it along the fixed ray to get $\gamma_{r, 1}$ based at $\alpha(r)$, its length

$$
L(r):=L\left(\gamma_{r, 1}\right)=L_{\infty}+O\left(r^{-\epsilon}\right)
$$

and its holonomy satisfies $\mid$ hol $-\mathrm{Id} \mid=O\left(r^{-1-\epsilon}\right)$. What is more, any loop based at $\alpha(r)$ with a length smaller than $\kappa r$ is generated by $\gamma_{r, 1}$ in the sense of Gromov.

Proof. In this case, $\left(B_{\kappa r}(\alpha(r)), r^{-2} g\right)$ converge to $B_{\kappa}((1,[\alpha])) \subset C(S(\infty))$ by Theorem 3.9. We may make $\kappa$ even smaller to apply Theorem 3.12. We get $\gamma_{r, 1}$ corresponding to $g_{r, 1}$ in Theorem 3.12. Then, any loop based at $\alpha(r)$ with a length smaller than $\kappa r$ is generated by $\gamma_{r, 1}$ in the sense of Gromov. There is ambiguity in choosing $\gamma_{r, 1}$. The same loop with the reverse direction would play the same role. However, we can choose them consistently so that they are the sliding of each other along the ray. By Theorem 3.12,

$$
\lim _{r \rightarrow \infty} \frac{L(r)}{r}=0 .
$$

So, the holonomy along the loop converges to the identity by Theorem 3.4. It follows that

$$
\mid \text { hol }-\mathrm{Id}|(r)=| \text { hol }-\mathrm{Id}\left|(\infty)-\int_{r}^{\infty}\right| \mathrm{hol}-\left.\mathrm{Id}\right|^{\prime} d t \leqslant 0+\int_{r}^{\infty} C L t^{-2-\epsilon} d t \leqslant O\left(r^{-\epsilon}\right)
$$

by the equation that || hol $-\left.\mathrm{Id}\right|^{\prime} \mid<C L r^{-2-\epsilon}$. Plug this back into the equation

$$
\left|L^{\prime}\right| \leqslant \mid \text { hol }-\mathrm{Id} \mid \text {. }
$$

We obtain

$$
\begin{aligned}
L(r) & =L\left(r_{0}\right)+\int_{r_{0}}^{r} L^{\prime}(t) d t \leqslant L\left(r_{0}\right)+\int_{r_{0}}^{r}|\mathrm{hol}-\mathrm{Id}| d t \\
& \leqslant L\left(r_{0}\right)+\int_{r_{0}}^{r} C t^{-\epsilon} d t=L\left(r_{0}\right)+C\left(r^{1-\epsilon}-r_{0}^{1-\epsilon}\right) .
\end{aligned}
$$

In turn, $\mid$ hol $-\mathrm{Id} \mid \leqslant O\left(r^{-2 \epsilon}\right), L \leqslant O\left(r^{1-2 \epsilon}\right)$. Through finite steps of iterations, we have

$$
L=L_{\infty}+O\left(r^{-\epsilon}\right) \quad \text { and } \quad \mid \text { hol }-\mathrm{Id} \mid \leqslant O\left(r^{-1-\epsilon}\right) .
$$


Claim. The limit length

$$
L_{\infty}=\lim _{t \rightarrow \infty} L_{t}>0 .
$$

Otherwise, because $L=O\left(r^{-\epsilon}\right)$, after the integration from infinity to $r$, we can easily obtain

$$
\mid \text { hol }-\mathrm{Id} \mid \leqslant O\left(r^{-1-2 \epsilon}\right)
$$

After a finite number of iterations, we have

$$
L=O\left(r^{-1-\epsilon}\right) \quad \text { and } \quad \mid \text { hol }-\mathrm{Id} \mid \leqslant O\left(r^{-2-2 \epsilon}\right) .
$$

Now, let

$$
f(r)=\sum_{k=0}^{\infty} \frac{2^{(2+\epsilon) k} r^{-k \epsilon}}{\epsilon(\epsilon+1) 2 \epsilon(2 \epsilon+1) \ldots k \epsilon(k \epsilon+1)} .
$$

Then,

$$
f^{\prime \prime}(r)=\left(\frac{1}{2} r\right)^{-2-\epsilon} f(r), \quad f(r)=1+O\left(r^{-\epsilon}\right), \quad f^{\prime}(r)=O\left(r^{-1-\epsilon}\right) .
$$

So, for any $R$ large enough, we have

$$
L(R)<R^{-1} f(R)
$$

and

$$
\left|L^{\prime}(R)\right|<R^{-1}\left|f^{\prime}(R)\right| \text {. }
$$

By the ODE comparison, we have $L(r)<R^{-1} f(r)$. Let $R$ goes to infinity. We see that $L(r)=0$. This is a contradiction. So, $L_{\infty}>0$.

TheOREM 3.14. In the ALG case, there are commutative geodesic loops $\gamma_{1}$ and $\gamma_{2}$ such that when we slide them along the fixed ray $\alpha$ to get $\gamma_{r, 1}$ and $\gamma_{r, 2}$ based at $\alpha(r)$, their length $L_{j}(r):=L\left(\gamma_{r, j}\right)=L_{\infty, j}+O\left(r^{-\epsilon}\right)$ and their holonomy satisfy $\mid$ hol $-\mathrm{Id} \mid=O\left(r^{-1-\epsilon}\right)$. What is more, any loop based at $\alpha(r)$ with a length smaller than $\kappa r$ is generated by $\gamma_{r, 1}$ and $\gamma_{r, 2}$ in the sense of Gromov.

Proof. We proceed as in the proof of Theorem 3.13. We get two loops, $\gamma_{r, 1}$ and $\gamma_{r, 2}$, based at $\alpha(r)$. In this case, the ambiguity is as large as $\operatorname{GL}(2, \mathbb{Z})$. In other words, $\gamma_{r, 1}$ and $\gamma_{r, 2}$ may jump to $\gamma_{r, 1}^{100} \gamma_{r, 2}^{99}$ and $\gamma_{r, 1}^{101} \gamma_{r, 2}^{100}$, respectively, after the sliding. Actually, $\mathrm{GL}(2, \mathbb{Z})$ is a noncompact group, so we cannot estimate the length of the geodesic loops obtained by sliding directly. However, we can still get the same conclusion from the fact that $\gamma_{r, 1}$ and $\gamma_{r, 2}$ commute and that they form a detectable angle.

Suppose that the manifold is flat. Then, the covering transforms corresponding to $\gamma_{r, 1}$ and $\gamma_{r, 2}$ are linear maps

$$
T_{1}(\mathbf{x})=\mathbf{a x}+\mathbf{b} \quad \text { and } \quad T_{2}(\mathbf{x})=\mathbf{A x}+\mathbf{B},
$$


where $\mathbf{a}, \mathbf{A} \in \mathrm{SU}(2), \mathbf{b}, \mathbf{B} \in \mathbb{C}^{2}$. So,

$$
\mathbf{x}=T_{1}^{-1} T_{2}^{-1} T_{1} T_{2}(\mathbf{x})=\mathbf{a}^{-1} \mathbf{A}^{-1} \mathbf{a A x}+\mathbf{a}^{-1} \mathbf{A}^{-1}((\mathbf{a}-\mathbf{I d}) \mathbf{B}+(\mathbf{I d}-\mathbf{A}) \mathbf{b}) .
$$

Note that by the construction, $|\mathbf{b}|<C|\mathbf{B}|$. On the manifold, we need to count the error caused by the curvature. So, actually

$$
|(\mathbf{a}-\mathbf{I d}) \mathbf{B}-(\mathbf{A}-\mathbf{I d}) \mathbf{b}| \leqslant C r^{-2-\epsilon}|\mathbf{b}||\mathbf{B}|^{2} \quad \text { and } \quad\left|\mathbf{a}^{-1} \mathbf{A}^{-1} \mathbf{a A}-\mathbf{I d}\right|<C r^{-2-\epsilon}|\mathbf{b}||\mathbf{B}| .
$$

Now, if $|\mathbf{a}-\mathbf{I d}|>r^{-1-\epsilon / 3}|\mathbf{b}|$, then

$$
|\mathbf{A}-\mathbf{I d}||\mathbf{b}| \geqslant|\mathbf{a}-\mathbf{I d}||\mathbf{B}|-C r^{-2-\epsilon}|\mathbf{b}||\mathbf{B}|^{2} .
$$

It follows that $|\mathbf{A}-\mathbf{I d}|>c \cdot r^{-1-\epsilon / 3}|\mathbf{B}|$ for some constant $c$. Thus, if $r$ is large enough, the two vectors $(\mathbf{A}-\mathbf{I d}) \mathbf{b}$ and $(\mathbf{a}-\mathbf{I d}) \mathbf{B}$ have almost the same angle because their difference has a much smaller length. Note that both $\mathbf{A}$ and $\mathbf{a}$ are very close to the identity, so $\mathbf{A}-\mathbf{I d}$ and $\mathbf{a}-\mathbf{I d}$ are almost $\log (\mathbf{A})$ and $\log (\mathbf{a})$, respectively. So, Theorem 3.12 is reduced to that $(\mathbf{a}-\mathbf{I d}, \mathbf{b})$ form a detectable angle with $(\mathbf{A}-\mathbf{I d}, \mathbf{B})$. Therefore, $\mathbf{A}-\mathbf{I d}$ and $\mathbf{a}-\mathbf{I d}$ also form a detectable angle because $(\mathbf{A}-\mathbf{I d}) \mathbf{b}$ has almost the same angle with $(\mathbf{a}-\mathbf{I d}) \mathbf{B}$.

Because the Lie algebra in $\mathfrak{s u}(2)$ is simply the cross product and all of the matrices are very close to the identity,

$$
|\mathbf{a}-\mathbf{I d}||\mathbf{A}-\mathbf{I d}|<C\left|\mathbf{a}^{-1} \mathbf{A}^{-1} \mathbf{a A}-\mathbf{I d}\right|<C r^{-2-\epsilon}|\mathbf{b}||\mathbf{B}| .
$$

This is a contradiction. So, $|\mathbf{a}-\mathbf{I d}| \leqslant r^{-1-\epsilon / 3}|\mathbf{b}|$. Similarly, $|\mathbf{A}-\mathbf{I d}| \leqslant r^{-1-\epsilon / 3}|\mathbf{B}|$.

We have proved that for $\gamma_{r, 1}$ and $\gamma_{r, 2}, \mid$ hol $-\mathrm{Id} \mid \leqslant r^{-1-\epsilon / 3} L$. For any loop with a length smaller than $\kappa r$, we have $\mid$ hol $-\mathrm{Id} \mid \leqslant C r^{-\epsilon / 3}$. When we slide $\gamma_{r, j}$ along the fixed ray toward infinity, the holonomy of the limiting loops must be trivial. The proof in Theorem 3.13 then implies our conclusion. Note that the ambiguity of choosing $\gamma_{r, j}$ can now be removed by requiring that they are the sliding of loops along $\alpha$.

THEOREM 3.15. In the ALH non-splitting case, there are commutative geodesic loops $\gamma_{1}, \gamma_{2}$, and $\gamma_{3}$ such that when we slide them along the fixed ray $\alpha$ to get $\gamma_{r, 1}, \gamma_{r, 2}$, and $\gamma_{r, 3}$ based at $\alpha(r)$, their length $L_{j}(r):=L\left(\gamma_{r, j}\right)=L_{\infty, j}+O\left(r^{-\epsilon}\right)$ and their holonomy satisfy $\mid$ hol $-\mathrm{Id} \mid=O\left(r^{-1-\epsilon}\right)$. What is more, any loop based at $\alpha(r)$ with a length smaller than $\kappa r$ is generated by $\gamma_{r, 1}, \gamma_{r, 2}$, and $\gamma_{r, 3}$ in the sense of Gromov.

Proof. We can proceed exactly in the same way as in Theorem 3.14. The only thing we need to prove is that $\gamma_{r, 2}$ commutes with $\gamma_{r, 3}$. It follows from the fact that the length of the commutator converges to zero, since the curvature and therefore the errors converge to zero as $r$ goes to zero. 


\subsection{From geodesic loops to Riemannian fiberation}

In [9], Cheeger, Fukaya, and Gromov first introduced the N-structure (i.e., nilpotent group fiberations of different dimensions patched together consistently). Torus is the simplest nilpotent group. In [34], Minerbe followed their method and improved the result for circle fiberations under a strong volume growth condition in the ALF case. In their papers, they all view $\mathbb{R}^{4-k} \times \mathbb{T}^{k}$ as the Gromov-Hausdorff approximation of $\mathbb{R}^{4-k}$. In this subsection, we also include the $\mathbb{T}^{k}$ factor in the analysis. Therefore, we are able to obtain a better estimate without any volume assumptions.

In the last subsection, we get geodesic loops $\gamma_{p, i}$ along a ray. They can be represented by $s \in[0,1] \mapsto \exp _{p}\left(s v_{i}(p)\right)$ for some vectors $v_{i}(p)$ in the tangent space of the base point $p$. When $p$ goes to infinity, the vectors $v_{i}(p)$ converge to some limits $v_{i} \in \mathbb{R}^{4}$. Actually, the difference between $v_{i}(p)$ and $v_{i}$ is $O\left(r^{-\epsilon}\right)$. Define the lattice $\Lambda=\bigoplus_{i=1}^{k} \mathbb{Z} v_{i}$ and the torus $\mathbb{T}^{k}=\left(\bigoplus_{i=1}^{k} \mathbb{R} v_{i}\right) / \Lambda$ with the induced metric. From the estimates in the last subsection and the estimates in the last paragraph of $\S 3.1$ (cf. [7, Proposition 2.3.1]), it is easy to see that, for $\sum_{i=1}^{k} a_{i} v_{i} \in \Lambda \cap B_{\kappa r(p)}$, the translation part of the Gromov product $\prod_{i=1}^{k} \gamma_{p, i}^{a_{i}}$ is $\sum_{i=1}^{k} a_{i} v_{i}$ with an error $O\left(r^{1-\epsilon}\right)$, whereas the holonomy is $O\left(r^{-\epsilon}\right)$. So, the lattice $\Lambda$ almost represents the geodesic loops whose lengths are smaller than $\kappa r(p)$.

By Proposition 3.3, Corollary 3.8, the estimates in Theorems 3.13-3.15, we can slide the geodesic loops $\gamma_{p, i}$ along a path within $B_{1.1 r}(o) \backslash B_{0.9 r}(o)$ to get geodesic loops $\gamma_{p, i}$ over the whole manifold $M$ except a compact set $K$. It satisfies all of the above properties. The choice of path is not unique, so after sliding along different paths, $\gamma_{p, i}$ may be different. However, all of the differences come from a change of basis in $\Lambda$. Locally, we may assume that $\gamma_{p, i}$ are well defined.

THEOREM 3.16. We can find a diffeomorphism from $B_{\kappa r}(p)$ to

$$
B_{\kappa r}(0) \times \mathbb{T}^{k} \subset \mathbb{R}^{4-k} \times \mathbb{T}^{k}
$$

such that $g$ is the pull-back of the flat metric plus $O^{\prime}\left(r^{-\epsilon}\right)$.

Proof. First of all, we look at the map exp: $\mathrm{T}_{p} \rightarrow M$. Any $q \in B_{\kappa r}(p)$ has lots of preimages. Choose one preimage $q_{0}$. Then, all of the other preimages are $\prod_{i=1}^{k} F_{i}^{a_{i}}\left(q_{0}\right)$, where $F_{i}$ are the covering transforms corresponding to $\gamma_{i}$, and $a_{i}$ are integers. We know that $\prod_{i=1}^{k} F_{i}^{a_{i}}\left(q_{0}\right)$ is actually $q_{0}+\sum_{i=1}^{k} a_{i} v_{i}$ with an error $O^{\prime}\left(r^{1-\epsilon}\right)$. Define

$$
f(q)=\pi_{\mathbb{T}^{k}} \frac{\sum \chi\left(\frac{10\left|\prod_{i=1}^{k} F_{i}^{a_{i}}\left(q_{0}\right)\right|}{\kappa r(p)}\right)\left(\prod_{i=1}^{k} F_{i}^{a_{i}}\left(q_{0}\right)-\sum_{i=1}^{k} a_{i} v_{i}\right)}{\sum \chi\left(\frac{10\left|\prod_{i=1}^{k} F_{i}^{a_{i}}\left(q_{0}\right)\right|}{\kappa r(p)}\right)} \in \mathbb{R}^{4-k} \times \mathbb{T}^{k},
$$


then it is independent of the choice of $q_{0}$. Roughly speaking, $f(q)$ is the weighted average of the projections of all of the preimages of $q_{0}$ to $\mathbb{R}^{4-k} \times \mathbb{T}^{k}$. It is easy to prove that using $f$, the metric $g$ is the pull-back of the flat metric plus $O^{\prime}\left(r^{-\epsilon}\right)$.

Lemma 3.17. We can find good covers $\left\{B_{\kappa r\left(p_{i}\right) / 2}\left(p_{i}\right)\right\}_{i \in I}$ such that $I$ can be divided into $I=I_{1} \cup \ldots \cup I_{N}$, and if $i, j \in I_{l}, l=1,2, \ldots, N$, then $B_{\kappa r\left(p_{i}\right)}\left(p_{i}\right) \cap B_{\kappa r\left(p_{j}\right)}\left(p_{j}\right)=\varnothing$.

Proof. This type of theorem was first proved in [9]. In our situation, we can choose maximal $\kappa 2^{l-1}$ nets in $B\left(2^{l+1}\right) \backslash B\left(2^{l}\right)$. Then, the volume comparison theorem implies the property.

THEOREM 3.18. Outside of a compact set $K$, there is a global fiberation and a $\mathbb{T}^{k}$ invariant metric $\tilde{g}=g+O^{\prime}\left(r^{-\epsilon}\right)$ with the curvature $O^{\prime}\left(r^{-2-\epsilon}\right)$.

Proof. By Lemma 3.17, we can first modify $i \in I_{1}$ and $j \in I_{2}$ so that they are compatible. Then, modify $i \in I_{1}, j \in I_{2}$, and $l \in I_{3}$ to make sure that they are compatible. After $N$ times, we are done. So, we start from a map

$$
f_{i j}: B_{\kappa r_{i}}\left(p_{i}\right) \times \mathbb{T}^{k} \longrightarrow B_{\kappa r_{j}}\left(p_{j}\right) \times \mathbb{T}^{k} .
$$

defined by

$$
f_{i j}(q, \theta)=\left(f_{i j}^{1}(q, \theta), f_{i j}^{2}(q, \theta)\right)=f_{j} \circ f_{i}^{-1}(q, \theta) .
$$

Average it and get $\tilde{f}_{i j}^{1}: B_{\kappa r_{i}}\left(p_{i}\right) \rightarrow B_{\kappa r_{j}}\left(p_{j}\right)$ by

$$
\tilde{f}_{i j}^{1}(q)=\frac{1}{\operatorname{Vol}\left(\mathbb{T}^{k}\right)} \int_{\mathbb{T}^{k}} f_{i j}^{1}(q, \theta) d \theta .
$$

From the higher derivative control, we know that the distance from the origin to

$$
f_{i j}^{2}(q, \theta)-f_{i j}^{2}(q, 0)-\theta \in \mathbb{T}^{k}
$$

is $O\left(r^{-\epsilon}\right)$. (Here we view $\mathbb{T}^{k}$ as an Abelian group.) For any $r$ large enough, we can lift it to $\mathbb{R}^{k}$ while keeping it $O\left(r^{-\epsilon}\right)$. Fix $q$, average it with respect to $\theta$, and then project it back to $\mathbb{T}^{k}$. We get a map $\tilde{f}_{i j}^{2}: B_{\kappa r_{i}}\left(p_{i}\right) \rightarrow \mathbb{T}^{k}$. Define

$$
\tilde{f}_{i j}: B_{\kappa r_{i}}\left(p_{i}\right) \times \mathbb{T}^{k} \longrightarrow B_{\kappa r_{j}}\left(p_{j}\right) \times \mathbb{T}^{k}
$$

by

$$
\tilde{f}_{i j}(q, \theta)=\left(\tilde{f}_{i j}^{1}(q), \theta+f_{i j}^{2}(q, 0)+\tilde{f}_{i j}^{2}(q)\right) .
$$

It is easy to see that $\left|\nabla^{m} \tilde{f}_{i j}\right|=O\left(r^{1-m-\epsilon}\right)$. We may glue the common part using $\tilde{f}_{i j}$. Now, there are two metrics $g_{i}^{\text {Flat }}$ and $g_{j}^{\text {Flat }}$. Choose a partition of unity $\chi_{i}+\chi_{j}=1$, with $\left|\nabla^{m} \chi_{i}\right|=O\left(r^{-m}\right)$. Let

$$
\tilde{g}=\chi_{i} g_{i}^{\text {Flat }}+\chi_{j} g_{j}^{\text {Flat }}
$$


It is a $\mathbb{T}^{k}$ invariant metric with $\left|\nabla^{m} \tilde{g}\right|=O\left(r^{-m-\epsilon}\right)$. Note that there are still two maps from $M$ to the gluing

$$
B_{\kappa r_{i}}\left(p_{i}\right) \times \mathbb{T}^{k} \cup_{\tilde{f}_{i j}} B_{\kappa r_{j}}\left(p_{j}\right) \times \mathbb{T}^{k}
$$

namely $\tilde{f}_{i j} \circ f_{i}$ and $f_{j}$. However, their distance is $O\left(r^{-\epsilon}\right)$. For any $r$ large enough, we can find out the unique $\tilde{g}$-minimal geodesic $\gamma$ satisfying $\gamma(0)=\tilde{f}_{i j} \circ f_{i}$ and $\gamma(1)=f_{j}$. Then, $\gamma\left(\chi_{j}\right)$ gives a new map from $M$ to

$$
B_{\kappa r_{i}}\left(p_{i}\right) \times \mathbb{T}^{k} \cup_{\tilde{f}_{i j}} B_{\kappa r_{j}}\left(p_{j}\right) \times \mathbb{T}^{k}
$$

Call that $\tilde{f}_{i} \cup \tilde{f}_{j}$.

In conclusion, we have a $\mathbb{T}^{k}$-invariant metric $h$ on

$$
B_{\kappa r_{i}}\left(p_{i}\right) \times \mathbb{T}^{k} \cup_{\tilde{f}_{i j}} B_{\kappa r_{j}}\left(p_{j}\right) \times \mathbb{T}^{k}
$$

and

$$
\tilde{f}_{i} \cup \tilde{f}_{j}: M \longrightarrow B_{\kappa r_{i}}\left(p_{i}\right) \times \mathbb{T}^{k} \cup_{\tilde{f}_{i j}} B_{\kappa r_{j}}\left(p_{j}\right) \times \mathbb{T}^{k},
$$

with both $\left|\nabla^{m} h\right|=O\left(r^{-m-\epsilon}\right)$ and $\left|\nabla^{m}\left(\tilde{f}_{i} \cup \tilde{f}_{j}\right)\right|=O\left(r^{1-m-\epsilon}\right)$.

After repeating everything for

$$
\left(B_{\kappa r_{i}}\left(p_{i}\right) \times \mathbb{T}^{k} \cup_{\tilde{f}_{i j}} B_{\kappa r_{j}}\left(p_{j}\right) \times \mathbb{T}^{k}, \tilde{g}, \tilde{f}_{i} \cup \tilde{f}_{j}\right) \quad \text { and } \quad\left(B_{\kappa r_{l}}\left(p_{l}\right) \times \mathbb{T}^{k}, g_{l}^{\text {flat }}, f_{l}\right),
$$

we can get a new big chart. After $N$ times, we are done.

TheOREM 3.19. Outside of $K$, there is a $\mathbb{T}^{k}$-fiberation $E$ over $C(S(\infty)) \backslash B_{R}$ and a standard $\mathbb{T}^{k}$ invariant metric $h$ such that, after the pull-back by some diffeomorphism, $h=g+O^{\prime}\left(r^{-\epsilon}\right)$.

Proof. The metric $\tilde{g}$ can be written as

$$
\sum_{i, j=1}^{4-k} a_{i j}(x) d x_{i} \otimes d x_{j}+\sum_{l=1}^{k}\left(d \theta_{l}+\sum_{i=1}^{4-k} \eta_{l i}(x) d x_{i}\right)^{2} .
$$

The curvature of $a_{i j}$ is $O^{\prime}\left(r^{-2-\epsilon}\right)$. By the result of Bando, Kasue, and Nakajima [3], there is a coordinate at infinity such that the difference between $a_{i j}$ and the flat metric on $C(S(\infty)) \backslash B_{R}$ is $O^{\prime}\left(r^{-\epsilon}\right)$. So, we may assume that $a_{i j}=\delta_{i j}$ without changing the condition $g=\tilde{g}+O^{\prime}\left(r^{-\epsilon}\right)$. Similarly, we may also replace $\eta_{l j}(x)$ with any standard connection form. As long as $\eta_{l j}$ is still $O^{\prime}\left(r^{-\epsilon}\right)$, we still have $h=g+O^{\prime}\left(r^{-\epsilon}\right)$. Therefore, we only need to classify the torus fiberations over $C(S(\infty)) \backslash B_{R}$ topologically, and give it a good-enough standard metric $h$. 
$\left(\right.$ ALF- $\left.A_{k}\right)$ When $S(\infty)=\mathbb{S}^{2}$, the circle fiberation must be orientable. It is determined by the Euler class $e$.

When $e=0$, we have the trivial product $\left(\mathbb{R}^{3} \backslash B_{R}\right) \times \mathbb{S}^{1}$ as our standard model.

When $e= \pm 1$, we have the Taub-NUT metric with mass $m \neq 0$. Let

$$
\begin{aligned}
& M_{+}=\left(\left\{\left(x_{1}, x_{2}, x_{3}\right): x_{1}^{2}+x_{2}^{2}+x_{3}^{2} \geqslant R^{2}\right\} \backslash\left\{\left(0,0, x_{3}\right): x_{3}<0\right\}\right) \times \mathbb{S}^{1}, \\
& M_{-}=\left(\left\{\left(x_{1}, x_{2}, x_{3}\right): x_{1}^{2}+x_{2}^{2}+x_{3}^{2} \geqslant R^{2}\right\} \backslash\left\{\left(0,0, x_{3}\right): x_{3}>0\right\}\right) \times \mathbb{S}^{1} .
\end{aligned}
$$

Identify $\left(x_{1}, x_{2}, x_{3}, \theta_{+}\right)$in $M_{+}$with $\left(x_{1}, x_{2}, x_{3}, \theta_{-}+\operatorname{sign}(m) \arg \left(x_{1}+i x_{2}\right)\right)$ in $M_{-}$. We get a manifold $M$.

Let $r=\sqrt{x_{1}^{2}+x_{2}^{2}+x_{3}^{2}}, V=1+2 m / r$, and

$$
\begin{aligned}
\eta & =4|m| d \theta_{+}+4 m \frac{\left(x_{3}-r\right)\left(x_{1} d x_{2}-x_{2} d x_{1}\right)}{2\left(x_{1}^{2}+x_{2}^{2}\right) r} \\
& =4|m| d \theta_{-}+4 m \frac{\left(x_{3}+r\right)\left(x_{1} d x_{2}-x_{2} d x_{1}\right)}{2\left(x_{1}^{2}+x_{2}^{2}\right) r} .
\end{aligned}
$$

Then, the Taub-NUT metric with mass $m$ outside of the ball $B_{R}(R \gg|m|)$ is

$$
d s^{2}=V d \mathbf{x}^{2}+V^{-1} \eta^{2},
$$

with

$$
d x_{1}=I^{*}\left(V^{-1} \eta\right)=J^{*} d x_{2}=K^{*} d x_{3} .
$$

There are lots of different conventions in the literature. We use the convention from [31], but we compute the explicit form of $\eta$ using the formulas in [23]. When $m>0$, LeBrun [31] proved that $M$ can be smoothly extended inside of $B_{R}$ and becomes biholomorphic to $\mathbb{C}^{2}$. For $m<0$, the metric is defined only outside of $B_{R}$, but it is enough for our purpose.

There is a natural $\mathbb{Z}_{|e|}$ action on the Taub-NUT metric by $\theta_{ \pm} \mapsto \theta_{ \pm}+2 \pi /|e|$ for $e=$ $\pm 1, \pm 2, \ldots$. The quotient of the Taub-NUT metric with positive mass $m$ by $\mathbb{Z}_{|e|}$ has Euler class $e<0$, The quotient of the Taub-NUT metric with negative mass $m$ by $\mathbb{Z}_{|e|}$ has Euler class $e>0$. Notice that the mass parameter $m$ is essentially a scaling parameter. Only the sign of $m$ determines the topology.

Usually, people let $k=-e-1$ and call that a standard ALF- $A_{k}$ metric.

$\left(\mathrm{ALF}-D_{k}\right)$ When

$$
S(\infty)=\mathbb{R} \mathbb{P}^{2}=\left\{\left(x_{1}, x_{2}, x_{3}\right) \in \mathbb{S}^{3}: x_{3} \geqslant 0\right\} /((\cos t, \sin t, 0) \sim(-\cos t,-\sin t, 0))
$$

topologically, the fiberation is the trivial fiberation over the disc following the identification of $(\cos t, \sin t, 0, \theta)$ with $(\cos (t+\pi), \sin (t+\pi), 0, f(t)-\theta)$. So $f(\pi)-f(0)=-2 e \pi$. The integer $e$ determines the topological type. 
When $e=0$, we have the trivial product $\left(\mathbb{R}^{3} \backslash B_{R}\right) \times \mathbb{S}^{1}$ after identifying $(\mathbf{x}, \theta)$ with $(-\mathbf{x},-\theta)$ as our standard model.

When $e$ is non-zero, it is the quotient of the Taub-NUT metric outside $B_{R}$ by the binary dihedral group

$$
D_{4|e|}=\left\langle\sigma, \tau: \sigma^{2|e|}=1, \sigma^{|e|}=\tau^{2}, \tau \sigma \tau^{-1}=\sigma^{-1}\right\rangle,
$$

which acts by $\sigma\left(\mathbf{x}, \theta_{ \pm}\right)=\sigma\left(\mathbf{x}, \theta_{ \pm}+\pi /|e|\right)$ and $\tau\left(\mathbf{x}, \theta_{+}\right)=\left(-\mathbf{x}, \theta_{-}=-\theta_{+}\right)$from $M_{+}$to $M_{-}$ with $\tau\left(\mathbf{x}, \theta_{-}\right)=\left(-\mathbf{x}, \theta_{+}=\pi-\theta_{-}\right)$from $M_{-}$to $M_{+}$. When the mass is positive, $e$ is negative. When the mass is negative, $e$ is positive.

Usually, people let $k=-e+2$ and call that a standard ALF- $D_{k}$ metric.

(ALG) When $S(\infty)=\mathbb{S}^{1}$, the topological type is determined by the monodramy. In other words, when we travel along $S(\infty)$, there is some rotation, but the lattice

$$
\Lambda=\mathbb{Z}\left|v_{1}\right| \oplus \mathbb{Z} \tau\left|v_{1}\right|
$$

is still invariant. So, we have the equation

$$
\left(\begin{array}{ll}
a & b \\
c & d
\end{array}\right)\left(\begin{array}{l}
1 \\
\tau
\end{array}\right)=\left(\begin{array}{cc}
e^{i \theta} & 0 \\
0 & e^{i \theta}
\end{array}\right)\left(\begin{array}{l}
1 \\
\tau
\end{array}\right)
$$

for some

$$
\left(\begin{array}{ll}
a & b \\
c & d
\end{array}\right) \in \mathrm{GL}(2, \mathbb{Z})
$$

So,

$$
0=\operatorname{det}\left(\begin{array}{cc}
a-e^{i \theta} & b \\
c & d-e^{i \theta}
\end{array}\right)=a d-b c-(a+d) e^{i \theta}+\left(e^{i \theta}\right)^{2} .
$$

Except for the case where $e^{i \theta}= \pm 1$, we have $\Delta=(a+d)^{2}-4(a d-b c)<0$. So, $a d-b c>0$ and it must be 1 to make sure that the matrix is invertible. So, $a+d=0$, or $a+d= \pm 1$. Thus, $e^{i \theta}$ must satisfy one of the following quadratic equations:

$$
x^{2}+x+1=0, \quad x^{2}-x+1=0, \quad \text { and } \quad x^{2}+1=0 .
$$

We can solve $e^{i \theta}$ accordingly:

$$
\frac{-1 \pm i \sqrt{3}}{2}=e^{2 i \pi / 3}, e^{4 i \pi / 3}, \quad \frac{1 \pm i \sqrt{3}}{2}=e^{i \pi / 3}, e^{5 i \pi / 3}, \quad \text { and } \quad \pm i=e^{i \pi / 2}, e^{3 i \pi / 2} .
$$

Therefore, the rotation angle $\theta=2 \pi \beta$ and the lattice $\Lambda=\mathbb{Z}\left|v_{1}\right| \oplus \mathbb{Z} \tau\left|v_{1}\right|$ are in the following list (we may replace $\tau$ with something like $\tau-1$, but that will not change the lattice at all): 
(Regular) $\operatorname{Im} \tau>0, \beta=1$.

(I $\left.\mathrm{I}_{0}^{*}\right) \operatorname{Im} \tau>0, \beta=\frac{1}{2}$.

(II) $\tau=e^{2 \pi i / 3}, \beta=\frac{1}{6}$.

(II*) $\tau=e^{2 \pi i / 3}, \beta=\frac{5}{6}$.

(III) $\tau=i, \beta=\frac{1}{4}$.

(III*) $\tau=i, \beta=\frac{3}{4}$.

(IV) $\tau=e^{2 \pi i / 3}, \beta=\frac{1}{3}$.

$\left(\mathrm{IV}^{*}\right) \tau=e^{2 \pi i / 3}, \beta=\frac{2}{3}$.

Note that they all correspond to Kodaira's classification of fibers of the elliptic surface in [28]! If we identify $(u, v)$ with $\left(e^{2 \pi i \beta} u, e^{-2 \pi i \beta} v\right)$ in the space

$$
\{(u, v): \arg u \in[0,2 \pi \beta],|u| \geqslant R\} \subset\left(\mathbb{C} \backslash B_{R}\right) \times \mathbb{C} /\left(\mathbb{Z}\left|v_{1}\right| \oplus \mathbb{Z} \tau\left|v_{1}\right|\right),
$$

we have the standard flat hyperkähler metric $h=\frac{1}{2} i(d u \wedge d \bar{u}+d v \wedge d \bar{v})$. Note that $\mathrm{SU}(2)$ is transitive, so we can choose the complex structure $a_{1} I+a_{2} J+a_{3} K$ properly so that $\bar{\partial}_{g}=\bar{\partial}_{h}+O\left(r^{-\epsilon}\right) \nabla_{h}$.

(ALH non-splitting) When $C(S(\infty))=\mathbb{R}_{+}, h$ can be simply chosen to be the product metric of $[R, \infty)$ and a flat 3 -torus.

\section{The construction of holomorphic functions}

In this section, we prove Theorems 1.2 and 1.3. Our goal in this section is to construct global holomorphic functions on gravitational instantons $M$ with prescribed growth orders. It is usually very hard to do so directly. However, it is much easier to construct holomorphic functions on the standard models $(E, h)$ first. Then, it can be pulled back to $(M, g)$, and cut off to obtain an almost holomorphic function $f$ on $M$. To get rid of the error, we can solve the $\bar{\partial}$ equation

$$
\bar{\partial} g=\bar{\partial} f
$$

for $g$ much smaller than $f$. If successful, then $f-g$ will be the required function. Unfortunately, it is hard for us to solve $g$ directly. So instead, we solve the following equation:

$$
-\left(\bar{\partial} \bar{\partial}^{*}+\bar{\partial}^{*} \bar{\partial}\right) \phi=\bar{\partial} f
$$

The order of $\bar{\partial}^{*} \phi$ and $\bar{\partial} \phi$ will be smaller than the order of $f$ if we solve $\phi$ properly. Notice that there is a covariant constant $(0,2)$ form $\omega^{-}$, so the harmonic $(0,2)$ form 
$\bar{\partial} \phi$ is essentially a harmonic function. Generally speaking, the order of the growth of harmonic functions on $M$ is the same as the harmonic functions on $E$. So, if we get $f$ from the smallest nonconstant harmonic function on $E$, we expect $\bar{\partial} \phi$ to be zero. Therefore, $f+\bar{\partial}^{*} \phi$ will be the required global holomorphic function on $M$.

To solve the Laplacian equation for $(0,1)$ forms, we need some elliptic estimates. The ALH case requires more care. To obtain a good estimate in the ALH case, we need to prove the exponential decay of curvature first. This is feasible after we develop some elliptic estimates for the Riemannian curvature tensor.

Therefore, in the first two subsections, we develop the elliptic estimates for tensors on a manifold $M$ asymptotic to the standard model. We would like to work on both forms and on the curvature tensors on general $M$, which may not be hyperkähler. Therefore, we always use the Bochner Laplacian $-\nabla^{*} \nabla$ to apply the Bochner techniques. For gravitational instantons, the Weitzenböck formula tells us that the Bochner Laplacian equals the operator $-\left(\bar{\partial} \bar{\partial}^{*}+\bar{\partial}^{*} \bar{\partial}\right)$ for functions and $(0,1)$ forms. Then, in the third subsection, we use the mentioned technique to construct global holomorphic functions on ALF and ALG instantons. In the fourth subsection, we use this estimate to prove the exponential decay of curvature of ALH instantons. This allows us to develop an elliptic estimate with exponential growth weights in the fifth subsection. In the sixth subsection, we use the same method to construct global holomorphic functions on ALH instantons In the last two subsections, we make use of the global holomorphic functions to prove our second and third main theorems.

Analysis in weighted Hilbert space is well studied, and perhaps some estimates in this section are already known to experts [24], [20], [33]. However, to avoid problems caused by subtle differences between different settings, we instead provide a self-contained proof.

\subsection{Weighted Hilbert space}

In this subsection, we do some technical preparations. We will use the following weighted Hilbert spaces. (Please notice the change in the meaning of $r$ as in the end of the second section.)

Definition 4.1. Define the $L_{\delta}^{2}$-norm of a tensor by

$$
\|\phi\|_{L_{\delta}^{2}}=\sqrt{\int_{M}|\phi|^{2} r^{\delta} d \mathrm{Vol} .}
$$

Let $L_{\delta}^{2}$ be the space of tensors with finite $L_{\delta}^{2}$-norm. Define $\nabla \phi=\psi$ in the distribution sense if, for any $\xi \in \mathrm{C}_{0}^{\infty}$, we have $\left(\phi, \nabla^{*} \xi\right)=(\psi, \xi)$. Let $H_{\delta}^{2}$ be the space of all tensors $\phi$ 
such that

$$
\phi \in L_{\delta}^{2}, \quad \nabla \phi \in L_{\delta+2}^{2}, \quad \text { and } \quad \nabla^{2} \phi \in L_{\delta+4}^{2} .
$$

We can define the norm in this weighted space by

$$
\|\phi\|_{H_{\delta}^{2}}=\sqrt{\int_{M}|\phi|^{2} r^{\delta} d \mathrm{Vol}+\int_{M}|\nabla \phi|^{2} r^{\delta+2} d \mathrm{Vol}+\int_{M}\left|\nabla^{2} \phi\right|^{2} r^{\delta+4} d \mathrm{Vol} .}
$$

The inner product is defined accordingly.

Proposition 4.2. For any $\delta$, we have that $H_{\delta}^{2}$ is a Hilbert space, and the space of compactly supported smooth tensors $\mathrm{C}_{0}^{\infty}$ is dense.

Proof. The map $\phi \mapsto \phi r^{\delta / 2}$ defines an isometry between $L_{\delta}^{2}$ and $L^{2}$. Because $L^{2}$ is complete, $L_{\delta}^{2}$ is also complete. Now, if $\left|\phi_{i}-\phi_{j}\right|_{H_{\delta}^{2}} \rightarrow 0$, then both

$$
\left|\phi_{i}-\phi_{j}\right|_{L_{\delta}^{2}} \quad \text { and } \quad\left|\nabla^{m} \phi_{i}-\nabla^{m} \phi_{j}\right|_{L_{\delta+2 m}^{2}}
$$

go to zero, $m=1$, 2. By completeness, $\phi_{i}$ converge to $\phi$ in $L_{\delta}^{2}$, and $\nabla \phi_{i}$ converge to $\psi$ in $L_{\delta+2}^{2}$. Now, pick any test tensor $\xi \in \mathrm{C}_{0}^{\infty}$,

$$
\left(\phi, \nabla^{*} \xi\right)=\lim _{i \rightarrow \infty}\left(\phi_{i}, \nabla^{*} \xi\right)=\lim _{i \rightarrow \infty}\left(\nabla \phi_{i}, \xi\right)=(\psi, \xi) .
$$

So, $\nabla \phi=\psi$ in the distribution sense. The second derivative is similar. So, $\phi_{i}$ converge to $\phi$ in $H_{\delta}^{2}$, too.

For the density, let $\chi_{R}=\chi(r / R)$. Then,

$$
\begin{aligned}
|\phi-\phi \chi(r / R)|_{H_{\delta}^{2}} \leqslant C \int_{M} & \left(\left|\left(1-\chi_{R}\right) \phi\right|^{2} r^{\delta}+\left|\left(1-\chi_{R}\right) \nabla \phi\right|^{2} r^{\delta+2}+\left|\nabla \chi_{R}\right||\phi|^{2} r^{\delta+2}\right. \\
& \left.+\left|\nabla^{2} \chi_{R} \phi\right|^{2} r^{\delta+4}+\left|\left(1-\chi_{R}\right) \nabla^{2} \phi\right|^{2} r^{\delta+4}+\left|\nabla \chi_{R} \nabla \phi\right| r^{\delta+4}\right)
\end{aligned}
$$

So, $\chi(r / R) \phi$ converge to $\phi$ in $H_{\delta}^{2}$ when $R$ goes to infinity, because $\left|\nabla \chi_{R}\right| \leqslant C / R$ and $\left|\nabla^{2} \chi_{R}\right| \leqslant C / R^{2}$. Now, the standard convolution method implies the density of $\mathrm{C}_{0}^{\infty}$.

Lemma 4.3. For any harmonic tensor $\phi$ in $H_{\delta}^{2}$ and any $r$ large enough,

$$
|\phi(y)| \leqslant C\|\phi\|_{H_{\delta}^{2}} r(y)^{-\delta / 2+k / 2-2} .
$$

When $-\frac{1}{2} \delta+\frac{1}{2} k-2<0$, we have $\phi=0$.

Proof. Given $y \in M$, suppose $r(y)=20 R$. Then, the ball $B_{2 R}(y) \subset M$ is asymptotic to $B_{2 R}(0) \times \mathbb{T}^{k} \subset \mathbb{R}^{4-k} \times \mathbb{T}^{k}$. Consider the covering space $\mathbb{R}^{4}$ of $\mathbb{R}^{4-k} \times \mathbb{T}^{k}$. If we apply Gilbarg and Trudinger's Theorem 9.20 in [16], we get

$$
|\phi|^{2}(y) \leqslant \frac{C}{\left|B_{2 R}(0)\right|} \int_{B_{2 R}(0)}|\phi|^{2} .
$$


So,

$$
|\phi(y)| \leqslant C\|\phi\|_{H_{\delta}^{2}} r(y)^{-\delta / 2+k / 2-2} .
$$

Now, the maximal principle implies the last result in the lemma because

$$
\Delta|\phi|^{2}=2|\nabla \phi|^{2} \geqslant 0
$$

At this point, we need a weighted $L^{2}$-estimate.

Lemma 4.4. For the standard ALF, ALG, or ALH metric in Theorem 3.19, suppose that $\phi$ is a smooth form supported in $B_{\widetilde{R}} \backslash B_{R}$. Then, as long as $R$ is large enough,

$$
\int_{E}\left|\nabla^{2} \phi\right|^{2} r^{\delta+4}+\int_{E}|\nabla \phi|^{2} r^{\delta+2} \leqslant C\left(\int_{E}|\Delta \phi|^{2} r^{\delta+4}+\int_{E}|\phi|^{2} r^{\delta}\right) .
$$

Proof. We only need to prove the same thing on $B_{\kappa r_{j}}\left(p_{j}\right) \subset E$ uniformly. It is enough to consider the covering $B_{\kappa r_{j}}(0) \subset \mathbb{R}^{4}$. Notice that $h$ is a flat metric plus $O^{\prime}\left(r^{-1}\right)$. Thus, we can simply use [16, Theorem 9.11].

\subsection{Elliptic estimates with polynomial growth weights}

In this subsection, we will prove the main estimate for tensors in the weighted Hilbert space with polynomial growth weights.

We started the estimate for functions on $\mathbb{R}^{d}$. Then, we extend this to $\mathbb{T}^{k}$ invariant tensors. We can improve it to general tensors on the standard fiberation $E$. Then, we can transfer that estimate back to any manifold $M$ asymptotic to the standard model. This main estimate allows us to prove the solvablity of the Bochner Laplacian equation for tensors.

THEOREM 4.5. Suppose that $f$ is a real smooth function on $\mathbb{R}^{d}(d=1,2,3, \ldots)$ supported in an annulus and that $\delta$ is not an integer. Then,

$$
\int_{\mathbb{R}^{d}}|f|^{2} r^{\delta} d \mathrm{Vol}<C \int_{\mathbb{R}^{d}}|\Delta f|^{2} r^{\delta+4} d \mathrm{Vol} .
$$

Proof. For the Laplacian on the standard sphere $\mathbb{S}^{d-1}$, it is well known it has eigenfunctions $\phi_{j, l}$ with eigenvalue $-j(d-2+j), l=1,2, \ldots, n_{j}$. (For $d=1$, all $n_{j}$ are zero, except $n_{0}=1$ and $\phi_{j, 1}=1$.) We write $f$ in terms of those eigenfunctions:

$$
f \sim \sum_{j=0}^{\infty} \sum_{l=1}^{n_{j}} f_{j, l}(r) \phi_{j, l}(\theta),
$$


where

$$
f_{j, l}(r)=\int_{\mathbb{S}^{d-1}} f(r, \theta) \phi_{j, l}(\theta) d \mathrm{Vol}
$$

Then,

$$
\begin{aligned}
\Delta f & \sim \sum_{j=0}^{\infty} \sum_{l=1}^{n_{j}}\left(f_{j, l}^{\prime \prime}+\frac{d-1}{r} f_{j, l}^{\prime}-\frac{j(d-2+j)}{r^{2}} f_{j, l}\right) \phi_{j, l}(\theta) \\
& =\sum_{j=0}^{\infty} \sum_{l=1}^{n_{j}} r^{-j-d+1}\left[r^{2 j+d-1}\left(r^{-j} f_{j, l}\right)^{\prime}\right]^{\prime} \phi_{j, l}(\theta) .
\end{aligned}
$$

From the integral by parts and the Cauchy-Schwartz inequality

$$
\left(\int_{0}^{\infty} g^{2} r^{\mu} d r\right)^{2}=\left(\frac{-2}{\mu+1} \int_{0}^{\infty} g g^{\prime} r^{\mu+1} d r\right)^{2} \leqslant \frac{4}{(\mu+1)^{2}} \int_{0}^{\infty} g^{2} r^{\mu} d r \int_{0}^{\infty}\left(g^{\prime}\right)^{2} r^{\mu+2} d r
$$

So we get the Hardy's inequality

$$
\int_{0}^{\infty} g^{2} r^{\mu} d r \leqslant \frac{4}{(\mu+1)^{2}} \int_{0}^{\infty}\left(g^{\prime}\right)^{2} r^{\mu+2} d r
$$

Therefore,

$$
\begin{aligned}
\int_{0}^{\infty} & f_{j, l}^{2} r^{\delta} r^{d-1} d r \\
& =\int_{0}^{\infty}\left(r^{-j} f_{j, l}\right)^{2} r^{\delta+2 j+d-1} d r \\
& \leqslant \frac{4}{(\delta+2 j+d)^{2}} \int_{0}^{\infty}\left[\left(r^{-j} f_{j, l}\right)^{\prime}\right]^{2} r^{\delta+2 j+d+1} d r \\
& =\frac{4}{(\delta+2 j+d)^{2}} \int_{0}^{\infty}\left[r^{2 j+d-1}\left(r^{-j} f_{j, l}\right)^{\prime}\right]^{2} r^{\delta-2 j-d+3} d r \\
& \leqslant \frac{16}{(\delta+2 j+d)^{2}(\delta-2 j-d+4)^{2}} \int_{0}^{\infty}\left(r^{-j-d+1}\left[r^{2 j+d-1}\left(r^{-j} f_{j, l}\right)^{\prime}\right]^{\prime}\right)^{2} r^{\delta+4} r^{d-1} d r
\end{aligned}
$$

Using the Fubini theorem and the Hilbert-Schmidt theorem (when $d=2$, we get exactly the Fourier series, so the Hilbert-Schmidt theorem is reduced to Parseval's identity), as long as $\delta$ is not an interger, we are done.

TheOrem 4.6. Suppose that $(E, h)$ is the product of $[R, \infty)$ and $\mathbb{T}^{3}$, and that $\phi$ is a smooth $\mathbb{T}^{3}$-invariant tensor supporte $B_{\widetilde{R}} \backslash B_{R}$. Then, as long as $\delta$ is not an integer, for $R$ large enough,

$$
\int_{E}|\phi|^{2} r^{\delta} d \mathrm{Vol}<C \int_{E}|\Delta \phi|^{2} r^{\delta+4} d \mathrm{Vol}
$$


Proof. Since the tangent bundle is trivial, the estimate of tensors is reduced to the estimate of their coefficients, which has been proved in Theorem 4.5.

TheOrem 4.7. Suppose that $(E, h)$ is the standard ALG metric as in Theorem 3.19 and that $\phi$ is a smooth $\mathbb{T}^{2}$-invariant tensor supported in $B_{\widetilde{R}} \backslash B_{R}$. Then, as long as $30 \delta$ is not an integer, for $R$ large enough,

$$
\int_{E}|\phi|^{2} r^{\delta} d \mathrm{Vol}<C \int_{E}|\Delta \phi|^{2} r^{\delta+4} d \mathrm{Vol}
$$

Proof. Let $\beta=m / n$. Then, it is enough to do the same estimate on the $n$-fold covering $\widetilde{E} \backslash B_{R}$ of $E \backslash B_{R}$. Note that $\widetilde{E} \backslash B_{R}$ is the isometric product of the $m$-fold covering of $\mathbb{C} \backslash B_{R}$ and $\mathbb{T}^{2}$. So, it is enough to prove Theorem 4.5 on the $m$-fold cover of $\mathbb{C} \backslash B_{R}$. If we write

$$
f \sim \sum_{j=-\infty}^{\infty} f_{j}(r) e^{i \theta j / m},
$$

where $\theta \in[0,2 m \pi]$, then all the works in the proof of Theorem 4.5 go through, except that we have to replace $j$ by $j / m$ there. So, as long as $m \delta$ is not an integer, we are done $(m=1,2,3,5)$.

TheOREM 4.8. Suppose that $(E, h)$ is the standard ALF metric as in Theorem 3.19 and that $\phi$ is a smooth $\mathbb{S}^{1}$-invariant tensor supported in $B_{\widetilde{R}} \backslash B_{R}$. Then, as long as $\delta$ is not an integer, for $R$ large enough,

$$
\int_{E}|\phi|^{2} r^{\delta} d \mathrm{Vol}<C \int_{E}|\Delta \phi|^{2} r^{\delta+4} d \mathrm{Vol}
$$

Proof. By Theorem 3.19, it is enough to consider the trivial product of $\mathbb{R}^{3}$ and $\mathbb{S}^{1}$ or the Taub-NUT metric with non-zero mass $m$. We use 1-forms as an example because the proof for general tensors is similar. In the trivial product case, we can write any form as $A d x_{1}+B d x_{2}+C d x_{3}+D d \theta$. In the remaining cases, any form can be written as $A d x_{1}+B d x_{2}+C d x_{3}+D \eta$. In each case, we get four functions on $\mathbb{R}^{3} \backslash B_{R}$ which can be filled in by zero on $B_{R}$ to get smooth functions on $\mathbb{R}^{3}$. So, we can apply Theorem 4.5 to them. Since the Taub-NUT metric is the flat metric with error $O^{\prime}\left(r^{-1}\right)$, while $\eta=d \theta+O^{\prime}\left(r^{-1}\right)$ locally, by Lemma 4.4, we can get our estimate as long as $R$ is large enough.

TheOREm 4.9. Suppose that $(E, h)$ is the standard ALF, ALG, or ALH non-splitting metric in Theorem 3.19 and that $\phi$ is a smooth tensor supported in $B_{\widetilde{R}} \backslash B_{R}$. Then, as long as $30 \delta$ is not an integer, for $R$ large enough,

$$
\int_{E}|\phi|^{2} r^{\delta} d \mathrm{Vol}<C \int_{E}|\Delta \phi|^{2} r^{\delta+4} d \mathrm{Vol} .
$$


Proof. First, we average $\phi$ on each $\mathbb{T}^{k}(k=1,2,3)$ to get an invariant tensor $\phi_{0}$. Then, we only need to get some estimates of the $\phi-\phi_{0}$ part. It is enough to prove that in each $B_{\kappa r_{i}}\left(p_{i}\right) \subset E$,

$$
\int_{B_{\kappa r_{i}}\left(p_{i}\right)}\left|\phi-\phi_{0}\right|^{2} d \mathrm{Vol}<C \int_{B_{\kappa r_{i}}\left(p_{i}\right)}\left|\Delta\left(\phi-\phi_{0}\right)\right|^{2} d \mathrm{Vol}
$$

for a uniform constant $C$ and any tensor $\phi$ supported in $B_{\kappa r_{i}}\left(p_{i}\right) \subset E$, because then we can use the partition of unity and move every error term to the left-hand side by Lemma 4.4. Again, we may cancel error terms and assume that the metric is flat. So, the estimate of forms is reduced to the estimate of functions that are the coefficients of the forms. The standard Poincaré inequality on torus implies that

$$
\begin{aligned}
\left(\int_{B_{\kappa r} \times \mathbb{T}^{k}}\left|f-f_{0}\right|^{2}\right)^{2} & \leqslant C\left(\int_{B_{\kappa r} \times \mathbb{T}^{k}}\left|\nabla_{\mathbb{T}^{k}}\left(f-f_{0}\right)\right|^{2}\right)^{2} \\
& \leqslant C\left(\int_{B_{\kappa r} \times \mathbb{T}^{k}}\left|\nabla\left(f-f_{0}\right)\right|^{2}\right)^{2} \\
& =C\left(\int_{B_{\kappa r} \times \mathbb{T}^{k}}\left(f-f_{0}\right) \Delta\left(f-f_{0}\right)\right)^{2} \\
& \leqslant C \int_{B_{\kappa r} \times \mathbb{T}^{k}}\left|f-f_{0}\right|^{2} \int_{B_{\kappa r} \times \mathbb{T}^{k}}\left|\Delta\left(f-f_{0}\right)\right|^{2},
\end{aligned}
$$

where $\nabla_{\mathbb{T}^{k}}$ means the partial derivative with respect to the fiber direction. So, we are done when $R$ is large enough.

Lemma 4.10. Let $X, Y$, and $Z$ be Banach spaces, $D: X \rightarrow Y, i: X \rightarrow Z$ be bounded linear operators, and $i$ be compact. Suppose that $\|\phi\|_{X} \leqslant C\left(\|D \phi\|_{Y}+\|i \phi\|_{Z}\right)$. Then, as long as $\operatorname{Ker} D=\{0\}$, we have $\|\phi\|_{X} \leqslant C\|D \phi\|_{Y}$.

Proof. If the estimate does not hold, then there are $\phi_{k}$ satisfying $\left\|\phi_{k}\right\|=1$, but $\left\|D \phi_{k}\right\| \rightarrow 0$. By the compactness of $i$, we know that $\left\|i \phi_{k}-i \phi_{l}\right\|_{Z} \rightarrow 0$. So,

$$
\left\|\phi_{k}-\phi_{l}\right\|_{X} \leqslant C\left(\left\|D \phi_{k}-D \phi_{l}\right\|_{Y}+\left\|i \phi_{k}-i \phi_{l}\right\|_{Z}\right) \rightarrow 0 \text {. }
$$

So, $\phi_{k} \rightarrow \phi_{\infty}$. Then, $D \phi_{k} \rightarrow D \phi_{\infty}, D \phi_{\infty}=0$, and $\phi_{\infty} \in \operatorname{Ker} D$, which is a contradiction.

THEOREM 4.11. Suppose that $M$ is asymptotic to the standard ALF, ALG or ALH non-splitting model. Then, for any tensor $\phi \in H_{\delta}^{2}(M)$, as long as $30 \delta$ is not an integer and $-\frac{1}{2} \delta-2+\frac{1}{2} k<0$, we have

$$
\|\phi\|_{H_{\delta}^{2}(M)}<C \int_{M}|\Delta \phi|^{2} r^{\delta+4} d \mathrm{Vol}
$$


Proof. It is enough to prove everything for $\mathrm{C}_{0}^{\infty}$. Note that

$$
\Delta_{g} \phi=\Delta_{h} \phi+O\left(r^{-\epsilon}\right)\left|\nabla^{2} \phi\right|+O\left(r^{-\epsilon-1}\right)|\nabla \phi|+O\left(r^{-\epsilon-2}\right)|\phi| .
$$

After applying Theorem 4.9 and Lemma 4.4, we know that the estimate holds as long as $\phi$ is zero inside a big-enough ball $B_{R}$. For general $\phi$, we can apply the estimate to the form $(1-\chi(r / R))^{2} \phi$. So,

$$
\begin{aligned}
\|\phi\|_{H_{\delta}^{2}(M)} & <C\left(\int_{M}|\Delta \phi|^{2} r^{\delta+4} d \mathrm{Vol}+\|\phi\|_{H^{2}\left(B_{2 R}\right)}\right) \\
& <C\left(\int_{M}|\Delta \phi|^{2} r^{\delta+4} d \mathrm{Vol}+\int_{B_{4 R}}|\phi|^{2}\right)
\end{aligned}
$$

by [16, Theorem 9.11]. By Lemma 4.10 and Rellich's lemma, it is enough to prove that Ker $\Delta=\{0\}$. This follows from Lemma 4.3.

TheOrem 4.12. Suppose that $30 \delta$ is not an integer and that $-\frac{1}{2} \delta-2+\frac{1}{2} k<0$. For any $\phi \in L_{-\delta}^{2}(M)$, there exists a tensor $\psi \in H_{-\delta-4}^{2}(M)$ such that $\Delta \psi=\phi$.

Proof. Consider the Laplacian operator $\Delta: L_{-\delta-4}^{2} \rightarrow L_{-\delta}^{2}$. The formal adjoint is $\Delta^{*} \phi=r^{\delta+4} \Delta\left(r^{-\delta} \phi\right)$. Apply Theorem 4.11 to $r^{-\delta} \phi$ :

$$
C^{-1}\left\|\Delta^{*} \phi\right\|_{L_{-\delta-4}^{2}} \leqslant\|\phi\|_{H_{-\delta}^{2}}=\left\|r^{-\delta} \phi\right\|_{H_{\delta}^{2}} \leqslant C\left\|\Delta^{*} \phi\right\|_{L_{-\delta-4}^{2}} .
$$

So $\Delta^{*}$ has a closed range. Now,

$$
\left|(\phi, \theta)_{L_{-\delta}^{2}}\right| \leqslant\|\phi\|_{L_{-\delta}^{2}}\|\theta\|_{L_{-\delta}^{2}} \leqslant C\|\phi\|_{L_{-\delta}^{2}}\left\|\Delta^{*} \theta\right\|_{L_{-\delta-4}^{2}} .
$$

So, $\Delta^{*} \theta \rightarrow(\phi, \theta)_{L_{-\delta}^{2}}$ defines a bound linear function in the range of $\Delta^{*}$. Using the Riesz representation theorem, there exists $\psi \in \operatorname{Im}\left(\Delta^{*}\right)$ such that $\left(\psi, \Delta^{*} \theta\right)_{L_{-\delta-4}^{2}}=(\phi, \theta)_{L_{-\delta}^{2}}$. Now, we get the theorem from the standard elliptic regularity theory.

\subsection{Holomorphic functions on ALF and ALG instantons}

After proving the main estimate in the last subsection, we are ready to prove the existence of global holomorphic functions on both ALF and ALG instatons. Our first theorem deals with the growth order of harmonic functions on $M$

TheOREm 4.13. Suppose $M$ is asymptotic to the standard ALF or ALG model. Given any harmonic function $f \in L_{\delta}^{2}(M)$ for some $\delta$, there exists an $\gamma$ such that $f$ is $O\left(r^{\gamma}\right)$ but not $o\left(r^{\gamma}\right)$. Also, when $C(S(\infty))=\mathbb{C}_{\beta}(\mathrm{ALG}), \beta \gamma$ must be an integer. When $C(S(\infty))=\mathbb{R}^{3}\left(\mathrm{ALF}-A_{k}\right), \gamma$ must be an integer. When $C(S(\infty))=\mathbb{R}^{3} / \mathbb{Z}_{2}\left(\mathrm{ALF}-D_{k}\right), \gamma$ must be an even number. 
Proof. The function $f$ also belongs to $L_{\delta^{\prime}}^{2}(M)$ for other $\delta^{\prime}$. Without loss of generality, assume that $\delta$ is bigger than the supremum of those $\delta^{\prime}$ minus $\epsilon$. The supremum exists because of the vanishing part of Theorem 4.3. By Lemma 4.4, $f \in H_{\delta}^{2}(M)$. Cut off $f$ so that it vanishes inside of a large ball $B_{R}$. Move this function to $E$. Then, $\Delta\left(f(1-\chi(r / R)) \in L_{\delta+4+\epsilon}^{2}\right.$. Decompose $f(1-\chi(r / R))$ into $\mathbb{T}^{k}$-invariant part $f_{0}$ and the perpendicular part $f_{1}$.

Then, $f_{1}$ is much smaller than the growth rate of $f(1-\chi)$. Without loss of generality, we may assume that $f(1-\chi(r / R))$ is invariant.

Now again, we can transfer this invariant function to the tangent cone at infinity $C(S(\infty))$. When $C(S(\infty))=\mathbb{R}^{3} / \mathbb{Z}_{2}\left(\mathrm{ALF}-D_{k}\right)$, we get a function $\tilde{f}$ on its double cover $\mathbb{R}^{3}$ naturally. When $C(S(\infty))=\mathbb{C}_{\beta}$ (ALG), we get a function $\tilde{f}(z)$ on $\mathbb{C}=\mathbb{R}^{2}$ defined by $\tilde{f}=(f(1-\chi(r / R)))\left(z^{\beta}\right)$. Again, the growth rate of $\Delta(\tilde{f})$ is at most the growth rate of $\tilde{f}$ minus 2 then minus $\epsilon$, so we can find out a function $\psi$ whose growth rate is the rate of $\tilde{f}$ minus $\epsilon$ such that $\Delta \psi=\Delta(\tilde{f})$. So, $\tilde{f}-\psi$ becomes a harmonic function on $\mathbb{R}^{3}$ or $\mathbb{R}^{2}$. The gradient estimate implies that after taking derivatives for some times, we get zero. In other words, $\tilde{f}-\psi$ must be a polynomial, so the growth rate must be an integer. For the $C(S(\infty))=\mathbb{R}^{3} / \mathbb{Z}_{2}\left(\mathrm{ALF}-D_{k}\right)$ case, we may replace $\psi(x)$ by $\frac{1}{2}(\psi(x)+\psi(-x))$, so that it is invariant under the $\mathbb{Z}_{2}$ action. So, the polynomial must have an even degree.

Now we can prove the existence of a global holomorphic function on any ALG gravitational instanton.

THEOREM 4.14. There exists a global holomorphic function on any ALG gravitational instanton $M$ such that any fiber far enough is biholomorphic to a complex torus.

Proof. In this case, $k=2$. By Theorem 3.19, the metric near infinity is asymptotic to the elliptic surface $(E, h)$. For $(E, h), u^{1 / \beta}$ is a well-defined holomorphic function outside $B_{R}$. Now, if we pull back $u^{1 / \beta}$ from the elliptic surface, cut it off, and fill it in with zero inside $K$, we obtain a function $f$ satisfying

$$
\bar{\partial}_{g} f=\phi=O\left(r^{1 / \beta-1-\epsilon}\right) .
$$

Pick any small positive number $\delta \in(\max \{-2,2 / \beta-2 \epsilon\}, 2 / \beta-\epsilon)$ such that $30 \delta$ is not an integer. Thus, $\phi \in L_{-\delta}^{2}$. By Theorem 4.12, there exists $\psi \in H_{-\delta-4}^{2}$ such that

$$
\phi=\Delta \psi=-\left(\bar{\partial}^{*} \bar{\partial}+\bar{\partial} \bar{\partial}^{*}\right) \psi
$$

in the distribution sense. Elliptic regularity implies that $\psi$ is a smooth $(0,1)$ form. Take $\bar{\partial}$ on both sides of this equation. Notice that $\bar{\partial} \phi=0$. Thus,

$$
0=-\bar{\partial} \bar{\partial}^{*}(\bar{\partial} \psi)=\Delta(\bar{\partial} \psi) .
$$


By Lemma 4.3, $\bar{\partial} \psi=O\left(r^{1 / \beta-\epsilon / 2}\right)$. We can write this $(0,2)$ form as $\xi \omega^{+}$, where $\omega^{+}$is the parallel $(0,2)$ form. Then, $\xi$ is a harmonic function. By Theorem 4.13, $\xi$ is a constant. Therefore, $\bar{\partial}\left(f+\bar{\partial}^{*} \psi\right)=0$, so $f+\bar{\partial}^{*} \psi$ is a global holomorphic function. After analyzing the growth rate, we can also show that $\left|d \bar{\partial}^{*} \psi\right| \ll|d f|$ for any large $r$. So, the fiber far from origin is a compact Riemann surface with genus 1 . It must be a complex torus by the uniformization theorem.

Similarly, we can prove the following.

TheOREM 4.15. There exists a global holomorphic function on any ALF- $D_{k}$ gravitational instanton $M$.

Proof. The instanton $M$ is asymptotic to a fiberation over $\mathbb{R}^{3} / \mathbb{Z}_{2}=\mathbb{R}^{3} /(\mathbf{x} \sim-\mathbf{x})$. The function $\left(x_{2}+i x_{3}\right)^{2}$ is well defined over $E$. The proof of the last theorem will produce a global holomorphic function in the ALF- $D_{k}$ case.

The existence of a global holomorphic function on any ALF- $A_{k}$ gravitational instanton $M$ can also be proved the same way. Actually, Minerbe had a simpler proof in [35]. It is an essential step in his classification of ALF- $A_{k}$ instantons.

\subsection{Exponential decay of curvature of ALH instantons}

For ALH non-splitting instantons, there is a self-improvement forcing the curvature to decay exponentially. Therefore, the metric must converge to the flat one exponentially.

Proposition 4.16. If the Ricci curvature is zero, then

$$
\Delta R_{i j k l}=Q(\mathrm{Rm})
$$

Proof. We have

$$
\Delta R_{i j k l}=R_{i j k l, m}{ }^{m}=-R_{i j l m, k}{ }^{m}-R_{i j m k, l}{ }^{m}=-R_{i j l m}{ }_{k}{ }_{k}-R_{i j m k}{ }^{m}{ }_{l}+Q(\mathrm{Rm}) .
$$

By Bianchi identity and the vanishing of the Ricci curvature,

$$
R_{i j l m}{ }^{m}=R_{l m i j}{ }^{m}=-R_{l m j}{ }^{m}{ }_{, i}-R_{l m}{ }^{m}{ }_{i, j}=0 .
$$

Similarly,

$$
R_{i j m k}{ }^{, m}=0
$$

Then, we reach the conclusion.

THEOREM 4.17. In the ALH non-splitting case, there exists a constant $\mu$ such that the Riemannian curvature at $p$ is bounded by $C e^{-\mu r(p)}$. 
Proof. Pull back the Riemannian curvature tensor of $g$ to $\left([R, \infty) \times \mathbb{T}^{3}, h\right)$, where $h$ is the standard flat metric, we get a tensor $\mathbf{T}$ satisfying the equation $\mathbf{D T}=0$, where $\mathbf{D}=\mathbf{A}^{i j} \nabla_{i} \nabla_{j}+\mathbf{B}^{i} \nabla_{i}+\mathbf{C}$ is a tensor-valued, second-order elliptic operator such that

$$
\left|\mathbf{A}^{i j}-\delta^{i j} \mathbf{I d}\right| \leqslant C r^{-\epsilon}, \quad\left|\mathbf{B}^{i}\right| \leqslant C r^{-1-\epsilon}, \quad|\mathbf{C}| \leqslant C r^{-2-\epsilon} .
$$

By Theorem 3.1,

$$
|\mathbf{T}|=O\left(r^{-2-\epsilon}\right), \quad|\nabla \mathbf{T}|=O\left(r^{-3-\epsilon}\right), \quad\left|\nabla^{\mathbf{2}} \mathbf{T}\right|=O\left(r^{-4-\epsilon}\right),
$$

so $\mathbf{T} \in H_{\delta}^{2}$ for all $\delta<3+2 \epsilon$. By Theorem 4.11 and the interior $L^{2}$ estimate (cf. [16, Theorem 9.11]), for any $R$ large enough,

$$
\begin{aligned}
\int_{[R+2, \infty) \times \mathbb{T}^{3}}|\mathbf{T}|^{2} & \leqslant \int_{[R, \infty) \times \mathbb{T}^{3}}(r-R)^{\epsilon}(1-\chi(r-R))^{2}|\mathbf{T}|^{2} \\
& \leqslant C \int_{[R, \infty) \times \mathbb{T}^{3}}(r-R)^{\epsilon+4}|\mathbf{D}((1-\chi(r-R)) \mathbf{T})|^{2} \\
& \leqslant C\|\mathbf{T}\|_{H^{2}\left([R+1, R+2] \times \mathbb{T}^{3}\right)}^{2} \\
& \leqslant C \int_{[R, R+3] \times \mathbb{T}^{3}}|\mathbf{T}|^{2} .
\end{aligned}
$$

So

$$
\int_{[R, \infty) \times \mathbb{T}^{3}}|\mathbf{T}|^{2} \geqslant\left(1+\frac{1}{C}\right) \int_{[R+3, \infty) \times \mathbb{T}^{3}}|\mathbf{T}|^{2} .
$$

In other words, the Riemannian curvature decays exponentially in the $L^{2}$ sense. The improvement to $L^{\infty}$ bound is simply Gilbarg and Trudinger's Theorem 9.20 in [16].

From this better control of curvature, the holonomy of the loops $\gamma_{r, i}$ in Theorem 3.15 can be improved to $|\mathrm{hol}-\mathrm{Id}|<C e^{-\mu r}$. Therefore, we are able to prove the following theorem.

THEOREM 4.18. For any ALH non-splitting gravitational instanton $(M, g)$, there exist a positive number $\mu$, a compact subset $K \subset M$, and a diffeomorphism

$$
\Phi:[R, \infty) \times \mathbb{T}^{3} \longrightarrow M \backslash K
$$

such that

$$
\left|\nabla^{m}\left(\Phi^{*} g-h\right)\right|_{h} \leqslant C(m) e^{-\mu r}
$$

for any $m=0,1,2, \ldots$, where $h=d r^{2} \oplus h_{1}$ for some flat metric $h_{1}$ on $\mathbb{T}^{3}$. 


\subsection{Elliptic estimates with exponential growth weights}

In this subsection, we are trying to prove the elliptic estimates for weighted Hilbert spaces with exponential growth weights.

We first look at the Laplacian operator on $\mathbb{T}^{3}=\mathbb{R}^{3} / \Lambda$. We define the dual lattice $\Lambda^{*}$ by

$$
\Lambda^{*}=\left\{\lambda \in \mathbb{R}^{3}:\langle\lambda, v\rangle \in \mathbb{Z} \text { for all } v \in \Lambda\right\} .
$$

Then, $\Delta$ has eigenvalues $-4 \pi^{2}|\lambda|^{2}$ with eigenvectors $e^{2 \pi i\langle\lambda, \theta\rangle}$ for all $\lambda \in \Lambda^{*}$. We call $\delta$ critical if $\delta=4 \pi|\lambda|$ for some $\lambda \in \Lambda^{*}$. So, Theorem 4.5 is replaced by the following theorem on $[R, \infty) \times \mathbb{T}^{3}$.

THEOREM 4.19. Suppose that $f$ is a real smooth function on $[0, \infty) \times \mathbb{T}^{3}$ supported in $\left[R, R^{\prime}\right] \times \mathbb{T}^{3}$, and that $\delta$ is not critical. Then,

$$
\int_{[0, \infty) \times \mathbb{T}^{3}}|f|^{2} e^{\delta r} d \mathrm{Vol}<C \int_{[0, \infty) \times \mathbb{T}^{3}}|\Delta f|^{2} e^{\delta r} d \mathrm{Vol} .
$$

Proof. We write $f$ in terms of its Fourier series:

$$
f \sim \sum_{\lambda \in \Lambda^{*}} f_{\lambda}(r) e^{2 \pi i\langle\lambda, \theta\rangle} .
$$

Then,

$$
\begin{aligned}
\Delta f & \sim \sum_{\lambda \in \Lambda^{*}}\left(f_{\lambda}^{\prime \prime}(r)-4 \pi^{2}|\lambda|^{2} f_{\lambda}(r)\right) e^{2 \pi i\langle\lambda, \theta\rangle} \\
& =\sum_{\lambda \in \Lambda^{*}}\left(\frac{d}{d r}-2 \pi|\lambda|\right)\left(\frac{d}{d r}+2 \pi|\lambda|\right) f_{\lambda}(r) e^{2 \pi i\langle\lambda, \theta\rangle} .
\end{aligned}
$$

This time the Hardy's inequality is

$$
\int_{0}^{\infty} g^{2} e^{\nu r} d r \leqslant \frac{4}{\nu^{2}} \int_{0}^{\infty}\left(g^{\prime}\right)^{2} e^{\nu r} d r
$$

Therefore,

$$
\begin{aligned}
\int_{0}^{\infty} f_{\lambda}^{2} e^{\delta r} d r & =\int_{0}^{\infty}\left(e^{2 \pi|\lambda| r} f_{\lambda}\right)^{2} e^{(\delta-4 \pi|\lambda|) r} d r \\
& \leqslant \frac{4}{(\delta-4 \pi|\lambda|)^{2}} \int_{0}^{\infty}\left[\left(e^{2 \pi|\lambda| r} f_{\lambda}\right)^{\prime}\right]^{2} e^{(\delta-4 \pi|\lambda|) r} d r \\
& =\frac{4}{(\delta-4 \pi|\lambda|)^{2}} \int_{0}^{\infty}\left[\left(\frac{d}{d r}+2 \pi|\lambda|\right) f_{\lambda}\right]^{2} e^{\delta r} d r \\
& \leqslant \frac{16}{(\delta+4 \pi|\lambda|)^{2}(\delta-4 \pi|\lambda|)^{2}} \int_{0}^{\infty}\left[\left(\frac{d}{d r}-2 \pi|\lambda|\right)\left(\frac{d}{d r}+2 \pi|\lambda|\right) f_{\lambda}(r)\right]^{2} e^{\delta r} d r
\end{aligned}
$$

So, as long as $\delta$ is not critical, we are done. 
Now we define $\underline{L}_{\delta}^{2}$ by

$$
\|\phi\|_{\underline{L}_{\delta}^{2}}=\int_{M}|\phi|^{2} e^{\delta r} d \mathrm{Vol}
$$

and $\underline{H}_{\delta}^{2}$ by

$$
\|\phi\|_{\underline{H}_{\delta}^{2}}=\sqrt{\int_{M}|\phi|^{2} e^{\delta r} d \mathrm{Vol}+\int_{M}|\nabla \phi|^{2} e^{\delta r} d \mathrm{Vol}+\int_{M}\left|\nabla^{2} \phi\right|^{2} e^{\delta r} d \mathrm{Vol} .}
$$

Then, Theorem 4.12 is replaced by the following.

TheOREM 4.20. Suppose $\delta$ is not critical and $\delta<0$. For any $\phi \in \underline{L}_{\delta}^{2}$, there exists a tensor $\psi \in \underline{H}_{\delta}^{2}$ such that $\Delta \psi=\phi$.

\subsection{Holomorphic functions on ALH instantons}

To go through all the steps in the ALF and ALG cases, we first need to control the growth rate of harmonic functions.

Lemma 4.21. Let $(N, h)$ be a smooth manifold such that, outside of a compact set, it is exactly $[R, \infty) \times \mathbb{T}^{3}$ with the flat metric. Then, any smooth function $u$ on $N$ harmonic outside of a large-enough ball with at most exponential growth rate can be written as linear combinations of $1, r, e^{2 \pi|\lambda| r} e^{2 \pi i\langle\lambda, \theta\rangle}$, and an exponential decay function, where $r$ and $\theta$ are the coordinate functions on $[R, \infty) \times \mathbb{T}^{3}$ pulled back by the diffeomorphism.

Proof. Write $u$ as its Fourier series

$$
\sum_{\lambda \in \Lambda^{*}} u_{\lambda}(r) e^{2 \pi i\langle\lambda, \theta\rangle}
$$

Then, $u_{\lambda}^{\prime \prime}=4 \pi^{2}|\lambda|^{2} u_{\lambda}$. So,

$$
u \sim a_{0}+b_{0} r+\sum_{\lambda \in \Lambda^{*} \backslash\{0\}} a_{\lambda} e^{2 \pi|\lambda| r} e^{2 \pi i\langle\lambda, \theta\rangle}+\sum_{\lambda \in \Lambda^{*} \backslash\{0\}} b_{\lambda} e^{-2 \pi|\lambda| r} e^{2 \pi i\langle\lambda, \theta\rangle} .
$$

By Parserval's identity, the growth condition of $u$ implies that the first sum has finite terms. For the second sum $U$, Parseval's identity again implies that $\int_{[R, R+1] \times \mathbb{T}^{3}}|U|^{2}$ decays exponentially. By [16, Theorem 9.20], we have that $U$ also decays exponentially in the $L^{\infty}$ sense.

Now, we can still find the global holomorphic function on ALH non-splitting instanton $(M, g)$. 
THEOREM 4.22. In the ALH non-splitting case, there exist $\left(a_{1}, a_{2}, a_{3}\right) \in \mathbb{S}^{2}$ and a global holomorphic function with respect to $a_{1} I+a_{2} J+a_{3} K$ on $M$ such that any fiber far enough is biholomorphic to a complex torus.

Proof. As before, let

$$
[R, \infty) \times \mathbb{T}^{3}=\left\{(r, \theta): r \geqslant R \text { and } \theta=\left(\theta_{1}, \theta_{2}, \theta_{3}\right) \in \mathbb{R}^{3} / \Lambda\right\}
$$

Let $\Lambda^{*}$ be the dual lattice. Choose $\lambda \in \Lambda^{*} \backslash\{0\}$ with minimal length. Choose $\left(a_{1}, a_{2}, a_{3}\right) \in$ $\mathbb{S}^{2}$ such that

$$
\left(a_{1} I^{*}+a_{2} J^{*}+a_{3} K^{*}\right) d r=-\frac{\lambda_{1} d \theta_{1}+\lambda_{2} d \theta_{2}+\lambda_{3} d \theta_{3}}{|\lambda|} .
$$

The function $e^{2 \pi|\lambda| r} e^{2 \pi i\langle\lambda, \theta\rangle}$ is holomorphic with respect to $a_{1} I+a_{2} J+a_{3} K$. The growth rate of this function is exactly $O\left(e^{2 \pi|\lambda| r}\right)$.

Now, we pull back this function from $[R, \infty) \times \mathbb{T}^{3}$ to $M$, cut it off, and fill it in with zero inside $K$ so that we obtain a function $f$ satisfying

$$
\bar{\partial}_{g} f=\phi=O\left(e^{(2 \pi|\lambda|-\mu) r}\right)
$$

where $\mu$ is the constant in Theorem 4.17. So, $\phi \in \underline{L}_{-2 \delta}^{2}$ for any non-critical positive number $\delta \in(2 \pi|\lambda|-\mu, 2 \pi|\lambda|)$. By Theorem 4.20, there exists $\psi \in \underline{H}_{-2 \delta}^{2}$ satisfying

$$
\phi=\Delta \psi=-\left(\bar{\partial}^{*} \bar{\partial}+\bar{\partial} \bar{\partial}^{*}\right) \psi
$$

in the distribution sense. Elliptic regularity implies that $\psi$ is a smooth $(0,1)$ form. As before, $\bar{\partial} \psi=\xi \omega^{+}$is a harmonic $(0,2)$ form, so $\xi$ is a harmonic function of order $O\left(e^{\delta r}\right)$.

Now, we use a cutoff function and the diffeomorphism to average $g$ and the pull-back of $h$. We get a smooth metric $g^{\prime}$ on $M$, which is identically the pull-back of $h$ outside a very big ball. Now let $\nu$ be the infimum of positive $\nu^{\prime}$ such that $\xi$ is $O\left(e^{\nu^{\prime} r}\right)$. If $\nu>0$, then $\Delta_{g^{\prime}} \xi \in \underline{L}_{-2 \delta^{\prime}}^{2}$ for any positive $\nu>\delta^{\prime}>\nu-\mu$. It follows that there exists a function in $\underline{L}_{-2 \delta^{\prime}}^{2}$ whose Laplacian $\Delta_{g^{\prime}}$ is $\Delta_{g^{\prime}} \xi$. The difference of those two functions is a $g^{\prime}$-harmonic function. By Lemma 4.21, it must have an at most linear growth rate since the growth rate is below the first non-linear harmonic function. It follows that $\xi$ must be $O\left(e^{\delta^{\prime} r}\right)$, which is a contradiction. So, $\nu=0$. Therefore, $\xi$ is bounded by any exponential growth function.

So, $\Delta_{g^{\prime}} \xi$ decays exponentially. In particular, it is in $L_{1-\epsilon}^{2}$. By Theorem 4.12, we can find out a function in $H_{-3-\epsilon}^{2}$ whose $\Delta_{g^{\prime}}$ is $\Delta_{g^{\prime}} \xi$. Therefore, we know that $\xi$ is actually $O\left(r^{1+\epsilon}\right)$. Of course, $\bar{\partial} \psi=\xi \omega^{+}$has the same estimate. 
By Lemma 4.4, the harmonic $(0,1)$-form $\bar{\partial}^{*} \bar{\partial} \psi=\bar{\partial}\left(f+\bar{\partial}^{*} \psi\right)$ is $O\left(r^{\epsilon}\right)$, and its covariant derivative is $O\left(r^{-1+\epsilon}\right)$. The Weitzenböck formula implies that $-\nabla^{*} \nabla\left(\bar{\partial}^{*} \bar{\partial} \psi\right)=0$. Therefore,

$$
\int_{M}\left|\nabla\left(\bar{\partial}^{*} \bar{\partial} \psi\right)\right|^{2} \chi \leqslant \int_{M}\left|\bar{\partial}^{*} \bar{\partial} \psi\right|\left|\nabla\left(\bar{\partial}^{*} \bar{\partial} \psi\right)\right||\nabla \chi|
$$

for any smooth compactly supported $\chi$. Let $\chi=\chi(r-R)$ so that the right-hand side converges to zero. Therefore, $\bar{\partial}^{*} \bar{\partial} \psi$ is a covariant constant $(0,1)$-form. If this form is non-zero, it would be invariant under the holonomy of any loop. However, elements in $\mathrm{SU}(2)$ have no fixed point except the identity matrix, so $(M, g)$ must have trivial holonomy. Therefore, it is $\mathbb{R}^{4-k} \times \mathbb{T}^{k}$ with the flat metric. This contradicts our nonsplitting assumption. So, $\bar{\partial}^{*} \bar{\partial} \psi$ is actually identical to zero and $f+\bar{\partial}^{*} \psi$ is a global holomorphic function on $M$.

\subsection{Compactification of ALG and ALH non-splitting instantons}

In Theorems 4.14 and 4.22, we proved the existence of global holomorphic function $u$ in the ALG and ALH non-splitting cases such that any fiber far enough is biholomorphic to a complex torus. Notice that $d u$ is never zero on any fiber far enough. We define a holomorphic vector field $X$ by $\omega^{+}(Y, X)=d u(Y)$. Then, since

$$
X(u)=d u(X)=\omega^{+}(X, X)=0,
$$

we have that $X$ is well defined when it is restricted to each fiber far enough . On each fixed fiber far enough, there exists a unique holomorphic form $\phi$ such that $\phi(X)=1$. Locally,

$$
\omega^{+}=f(u, v) d u \wedge d v, \quad X=f^{-1}(u, v) \frac{\partial}{\partial v}, \quad \phi=f(u, v) d v .
$$

Notice that each fiber far enough is topologically a torus. So, actually, we can integrate the form $\phi$ to get a holomorphic function $v \in \mathbb{C} /\left(\mathbb{Z} \tau_{1}(u) \oplus \mathbb{Z} \tau_{2}(u)\right)$ up to a constant. We can fix this constant locally by choosing a holomorphic section of $u$ as the base point. Therefore, $M$ is biholomorphic to $(U \times \mathbb{C}) /\left((u, v) \sim\left(u, v+m \tau_{1}(u)+n \tau_{2}(u)\right)\right)$, where $\tau_{1}(u)$ and $\tau_{2}(u)$ are locally defined holomorphic functions. Actually, they are the integral of $\phi$ in the basis of $H_{1}$ of each fiber. This gives a local holomorphic torus fiberation.

Recall that there is a diffeomorphism from $M$ minus a large compact set to the standard fiberation. Denote the inverse image of the zero section by $s$. Note that $s$ is again a section outside of a large compact set because $d u$ differs with the standard one by a decaying error. Write $\bar{\partial} s$ as $e(u) d \bar{u} \otimes X$, and then $e$ is a function defined on the inverse of the punctured disc with polynomial growth rate. So, there is an at most polynomial 
growth function $E$ on the inverse of punctured disc such that

$$
\bar{\partial} E(u)=e(u) d \bar{u} .
$$

Now we apply the flow $-E(u) X$ to the section $s$ to get a holomorphic section $s_{0}$ on the neighborhood of infinity. We view $s_{0}$ as the zero section, and we know that $M$ minus a large compact set is biholomorphic to

$$
\left(\left(\mathbb{C} \backslash B_{R}\right) \times \mathbb{C}\right) /\left((u, v) \sim\left(u, v+m \tau_{1}(u)+n \tau_{2}(u)\right)\right)
$$

globally, where $\tau_{1}(u)$ and $\tau_{2}(u)$ are multi-valued holomorphic functions.

As proved in Kodaira's paper [28], there exists an (unique) elliptic fiberation $B$ over $B_{R^{-1}}$ with a section such that $B$ minus the central fiber $D$ is biholomorphic to

$$
\left(\left(B_{R^{-1}} \backslash\{0\}\right) \times \mathbb{C}\right) /\left((\tilde{u}, v) \sim\left(\tilde{u}, v+m \tau_{1}\left(\tilde{u}^{-1}\right)+n \tau_{2}\left(\tilde{u}^{-1}\right)\right)\right) .
$$

We can naturally identity points and get a compactification $\bar{M}$ of $M$. So, $\bar{M}$ is a compact complex surface with a meromorphic function $u=\tilde{u}^{-1}$. Now, since the subvariety of critical points $\{d u=0\}$ is a finite union of irreducible curves ( $u$ is of course constant on those irreducible curves) and points, we know that, except for finite critical values in $\mathbb{C P}^{1}$, any fiber of $u$ has no intersection with $\{d u=0\}$. Therefore, a generic fiber has genus 1 and must be an elliptic curve. In other words, $\bar{M}$ is a compact elliptic surface. In conclusion, we have proved the second main theorem.

\subsection{Twistor space of ALF- $D_{k}$ instantons}

On ALF- $D_{k}$ gravitational instantons, we have found quadratic growth holomorphic functions for each compatible complex structure. A natural question is the following: is there any relationship between those functions? Before going ahead, let us recall the definition of the twistor space of a hyperkähler manifold.

Definition 4.23. (Cf. [23]) Let $(M, g, I, J, K)$ be a hyperkähler manifold. Then, the twistor space $Z$ of $M$ is the product manifold $M \times \mathbb{S}^{2}$ equipped with an integrable complex structure

$$
\underline{I}=\left(\frac{1-\zeta \bar{\zeta}}{1+\zeta \bar{\zeta}} I-\frac{\zeta+\bar{\zeta}}{1+\zeta \bar{\zeta}} J+i \frac{\zeta-\bar{\zeta}}{1+\zeta \bar{\zeta}} K, I_{0}\right),
$$

where $\zeta \in \mathbb{C} \subset \mathbb{C} \cup\{\infty\}=\mathbb{C P}^{1}=\mathbb{S}^{2}$ is the coordinate function, and $I_{0}$ is the standard complex structure on $\mathbb{C P}^{1}$. 
Notice that our definition is different from [23] to correct a sign error. We will briefly rewrite pp. 554-557 of their paper with the correct sign.

Let $\phi$ be a $(1,0)$-form of $I$. Then, we have $I^{*} \phi=i \phi$, where $\left(I^{*} \phi\right)(X)=\phi(I X)$. Set $\theta=\phi+\zeta K^{*} \phi$, then

$$
(1+\zeta \bar{\zeta}) \underline{I}^{*} \theta=\left((1-\zeta \bar{\zeta}) I^{*}-(\zeta+\bar{\zeta}) J^{*}+i(\zeta-\bar{\zeta}) K^{*}\right) \theta=i(1+\zeta \bar{\zeta}) \theta
$$

because we have relationships like $J^{*} I^{*}=K^{*}$. (In [23], they thought $I^{*} J^{*}=K^{*}$ and caused a sign error.)

Now, if the form $\omega^{+}=\omega_{2}+i \omega_{3}$ can be written as

$$
\frac{1}{2} \omega^{+}=\sum_{i=1}^{n} \phi_{i} \wedge \phi_{n+i}
$$

for some $(1,0)$-forms of $I$, then we can define a form on the twistor space by

$$
\omega=2 \sum_{i=1}^{n}\left(\phi_{i}+\zeta K^{*} \phi_{i}\right) \wedge\left(\phi_{n+i}+\zeta K^{*} \phi_{n+i}\right)=\left(\omega_{2}+i \omega_{3}\right)+2 \zeta \omega_{1}-\zeta^{2}\left(\omega_{2}-i \omega_{3}\right) .
$$

It is a holomorphic section of the vector bundle $\Lambda^{2} T_{F}^{*} \otimes \mathcal{O}(2)$, where $F$ means the fiber of $Z$, which is diffeomorphic to $M$. We also have a real structure $\tau(p, \zeta)=(p,-1 / \bar{\zeta})$. It takes the complex structure $\underline{I}$ to its conjugate $-\underline{I}$. In [23], they proved the following theorem.

THEOREM 4.24. Let $Z^{2 n+1}$ be a complex manifold such that

(i) $Z$ is a holomorphic fiber bundle $\pi: Z \rightarrow \mathbb{C P}^{1}$ over the projective line;

(ii) The bundle admits a family of holomorphic sections each with a normal bundle isomorphic to $\mathbb{C}^{2 n} \otimes \mathcal{O}(1)$;

(iii) There exists a holomorphic section $\omega$ of $\Lambda^{2} T_{F}^{*} \otimes \mathcal{O}(2)$ defining a symplectic form on each fiber;

(iv) $Z$ has a real structure compatible with (i), (ii), and (iii) and inducing the antipodal map on $\mathbb{C P}^{1}$.

Then, the parameter space of real sections is a 4n-dimensional manifold with a natural hyperkähler metric for which $Z$ is the twistor space.

Return to the gravitational instantons for which $n$ in the above theorems equals 1 . Recall that we have found the holomorphic function on $M$ by modifying the pull-back of the standard function on the standard model. So, let us look at the standard model $(E, h, I, J, K)$ first. It is the quotient of the Taub-NUT metric outside a compact set by $D_{4|e|}$. Recall that the Taub-NUT metric is (cf. Theorem 3.19)

$$
d s^{2}=V d \mathbf{x}^{2}+V^{-1} \eta^{2},
$$


with

$$
d x_{1}=I^{*}\left(V^{-1} \eta\right)=J^{*} d x_{2}=K^{*} d x_{3}
$$

So,

$$
\left(\frac{1-\zeta \bar{\zeta}}{1+\zeta \bar{\zeta}} I^{*}-\frac{\zeta+\bar{\zeta}}{1+\zeta \bar{\zeta}} J^{*}+i \frac{\zeta-\bar{\zeta}}{1+\zeta \bar{\zeta}} K^{*}-i\right)\left(-2 \zeta d x_{1}-\left(1-\zeta^{2}\right) d x_{3}+i\left(1+\zeta^{2}\right) d x_{2}\right)=0
$$

Therefore, $\left(-x_{3}+i x_{2}-2 x_{1} \zeta-\left(-x_{3}-i x_{2}\right) \zeta^{2}\right)^{2}$ is a holomorphic function on the twistor space of $E$, so the holomorphic function on $M \times\{\zeta\} \in Z$ is asymptotic to

$$
\left(-x_{3}+i x_{2}-2 x_{1} \zeta-\left(-x_{3}-i x_{2}\right) \zeta^{2}\right)^{2}
$$

with error $O^{\prime}\left(r^{2-\epsilon}\right)$.

Notice that any harmonic function has an even-integer growth rate, so the holomorphic function is unique up to the adding of constants. We may fix this ambiguity by requiring the value at the fixed base point $o$ to be zero. We will prove that, after the modification, the holomorphic functions have a simple relationship.

Actually, we have a $I$-holomorphic $(\zeta=0)$ function $u_{1}+i v_{1}$ asymptotic to

$$
\left(-x_{3}+i x_{2}\right)^{2}=\left(x_{3}^{2}-x_{2}^{2}\right)-2 i x_{2} x_{3},
$$

$J$-holomorphic $(\zeta=-1)$ function $u_{2}+i v_{2}$ asymptotic to

$$
\left(2 x_{1}+2 i x_{2}\right)^{2}=4\left(x_{1}^{2}-x_{2}^{2}\right)+8 i x_{1} x_{2},
$$

and $K$-holomorphic $(\zeta=-i)$ function $u_{3}+i v_{3}$ asymptotic to

$$
\left(-2 x_{3}+2 i x_{1}\right)^{2}=4\left(x_{3}^{2}-x_{1}^{2}\right)-8 i x_{3} x_{1} .
$$

Notice that $u_{2}+u_{3}-4 u_{1}$ is a harmonic function asymptotic to zero, i.e., is $O^{\prime}\left(r^{2-\epsilon}\right)$, so it must be zero. Similarly, the harmonic function

$$
z(p, \zeta)=\left(u_{1}+i v_{1}\right)-\frac{1}{2}\left(v_{3}+i v_{2}\right) \zeta+\frac{1}{2}\left(u_{2}-u_{3}\right) \zeta^{2}+\frac{1}{2}\left(v_{3}-i v_{2}\right) \zeta^{3}+\left(u_{1}-i v_{1}\right) \zeta^{4}
$$

is asymptotic to

$$
\left(-x_{3}+i x_{2}-2 x_{1} \zeta-\left(-x_{3}-i x_{2}\right) \zeta^{2}\right)^{2}
$$

and therefore must be the holomorphic one. In conclusion, we have proved the following theorem.

Theorem 4.25. In the ALF- $D_{k}$ case, there exist six harmonic functions $u_{i}$ and $v_{i}$, with $4 u_{1}=u_{2}+u_{3}$, such that

$$
z(p, \zeta)=\left(u_{1}+i v_{1}\right)-\frac{1}{2}\left(v_{3}+i v_{2}\right) \zeta+\frac{1}{2}\left(u_{2}-u_{3}\right) \zeta^{2}+\frac{1}{2}\left(v_{3}-i v_{2}\right) \zeta^{3}+\left(u_{1}-i v_{1}\right) \zeta^{4}
$$

is a $\underline{I}$-holomorphic map from the twistor space of $M$ to the total space of the $\mathcal{O}(4)$ bundle over $\mathbb{C P}^{1}$.

There is a real structure on the $\mathcal{O}(4)$ bundle $(\zeta, \eta) \mapsto\left(-1 / \bar{\zeta}, \bar{\eta} / \bar{\zeta}^{4}\right)$. It is easy to see that the map $z$ commutes with the real structure. 


\section{References}

[1] Anderson, M. T., Kronheimer, P. B. \& LeBrun, C., Complete Ricci-flat Kähler manifolds of infinite topological type. Comm. Math. Phys., 125 (1989), 637-642.

[2] Атiуah, M. \& Hitchin, N., The Geometry and Dynamics of Magnetic Monopoles. M. B. Porter Lectures. Princeton Univ. Press, Princeton, NJ, 1988.

[3] Bando, S., Kasue, A. \& Nakajima, H., On a construction of coordinates at infinity on manifolds with fast curvature decay and maximal volume growth. Invent. Math., 97 (1989), 313-349.

[4] Biquard, O. \& Boalch, P., Wild non-abelian Hodge theory on curves. Compos. Math., 140 (2004), 179-204.

[5] Biquard, O. \& Minerbe, V., A Kummer construction for gravitational instantons. Comm. Math. Phys., 308 (2011), 773-794.

[6] Botlch, P., Symplectic manifolds and isomonodromic deformations. Adv. Math., 163 (2001), 137-205.

[7] Buser, P. \& Karcher, H., Gromov's Almost Flat Manifolds. Astérisque, 81. Société Mathématique de France, Paris, 1981.

[8] Cheeger, J. \& Colding, T. H., Lower bounds on Ricci curvature and the almost rigidity of warped products. Ann. of Math., 144 (1996), 189-237.

[9] Cheeger, J., Fukaya, K. \& Gromov, M., Nilpotent structures and invariant metrics on collapsed manifolds. J. Amer. Math. Soc., 5 (1992), 327-372.

[10] Cheeger, J. \& Gromoll, D., The splitting theorem for manifolds of nonnegative Ricci curvature. J. Differential Geometry, 6 (1971/72), 119-128.

[11] Cherkis, S. A. \& Hitchin, N. J., Gravitational instantons of type $D_{k}$. Comm. Math. Phys., 260 (2005), 299-317.

[12] Cherkis, S. A. \& Kapustin, A., Singular monopoles and gravitational instantons. Comm. Math. Phys., 203 (1999), 713-728.

[13] Colding, T.H., Ricci curvature and volume convergence. Ann. of Math., 145 (1997), $477-501$.

[14] Drees, G., Asymptotically flat manifolds of nonnegative curvature. Differential Geom. Appl., 4 (1994), 77-90.

[15] Fukaya, K., A boundary of the set of the Riemannian manifolds with bounded curvatures and diameters. J. Differential Geom., 28 (1988), 1-21.

[16] Gilbarg, D. \& Trudinger, N. S., Elliptic Partial Differential Equations of Second Order. Classics in Mathematics. Springer, Berlin-Heidelberg, 2001.

[17] Greene, R. E. \& Wu, H., Function Theory on Manifolds which Possess a Pole. Lecture Notes in Mathematics, 699. Springer, Berlin, 1979.

[18] Gromov, M., Almost flat manifolds. J. Differential Geometry, 13 (1978), 231-241.

[19] Haskins, M., Hein, H. J. \& Nordström, J., Asymptotically cylindrical Calabi-Yau manifolds. J. Differential Geom., 101 (2015), 213-265.

[20] Hausel, T., Hunsicker, E. \& Mazzeo, R., Hodge cohomology of gravitational instantons. Duke Math. J., 122 (2004), 485-548.

[21] Hawking, S. W., Gravitational instantons. Phys. Lett. A, 60 (1977), 81-83.

[22] Hein, H., Gravitational instantons from rational elliptic surfaces. J. Amer. Math. Soc., 25 (2012), 355-393.

[23] Hitchin, N. J., Karlhede, A., Lindström, U. \& RočEK, M., Hyper-Kähler metrics and supersymmetry. Comm. Math. Phys., 108 (1987), 535-589.

[24] Hörmander, L., An Introduction to Complex Analysis in Several Variables. North-Holland Mathematical Library, 7. North-Holland, Amsterdam, 1990. 
[25] Ivanov, I. T. \& RočEK, M., Supersymmetric $\sigma$-models, twistors, and the Atiyah-Hitchin metric. Comm. Math. Phys., 182 (1996), 291-302.

[26] Jost, J. \& Karcher, H., Geometrische Methoden zur Gewinnung von a-priori-Schranken für harmonische Abbildungen. Manuscripta Math., 40 (1982), 27-77.

[27] Kasue, A., A compactification of a manifold with asymptotically nonnegative curvature. Ann. Sci. École Norm. Sup., 21 (1988), 593-622.

[28] Kodaira, K., On compact analytic surfaces. II. Ann. of Math., 77 (1963), 563-626.

[29] Kronheimer, P. B., The construction of ALE spaces as hyper-Kähler quotients. J. Differential Geom., 29 (1989), 665-683.

[30] - A Torelli-type theorem for gravitational instantons. J. Differential Geom., 29 (1989), 685-697.

[31] LeBrun, C., Complete Ricci-flat Kähler metrics on $\mathbf{C}^{n}$ need not be flat, in Several Complex Variables and Complex Geometry, Part 2 (Santa Cruz, CA, 1989), Proc. Sympos. Pure Math., 52, pp. 297-304. Amer. Math. Soc., Providence, RI, 1991.

[32] Mashiko, Y., Nagano, K. \& Otsuka, K., The asymptotic cones of manifolds of roughly non-negative radial curvature. J. Math. Soc. Japan, 57 (2005), 55-68.

[33] Minerbe, V., A mass for ALF manifolds. Comm. Math. Phys., 289 (2009), 925-955.

[34] - On the asymptotic geometry of gravitational instantons. Ann. Sci. École Norm. Sup., 43 (2010), 883-924.

[35] — Rigidity for multi-Taub-NUT metrics. J. Reine Angew. Math., 656 (2011), 47-58.

[36] Ruh, E. A., Almost flat manifolds. J. Differential Geometry, 17 (1982), 1-14.

[37] SAKAI, H., Rational surfaces associated with affine root systems and geometry of the Painlevé equations. Comm. Math. Phys., 220 (2001), 165-229.

[38] Tian, G. \& Yau, S.-T., Complete Kähler manifolds with zero Ricci curvature. I. J. Amer. Math. Soc., 3 (1990), 579-609.

[39] YAu, S.-T., The role of partial differential equations in differential geometry, in Proceedings of the International Congress of Mathematicians (Helsinki, 1978), pp. 237-250. Acad. Sci. Fennica, Helsinki, 1980.

\section{Gao Chen}

Institute of Geometry and Physics University of Science and Technology of China

No. 96 Jinzhai Road

Hefei, 230026

China

chengao1@ustc.edu.cn

\author{
Xiuxiong Chen \\ Institute of Geometry and Physics \\ University of Science and Technology \\ of China \\ No. 96 Jinzhai Road \\ Hefei, 230026 \\ China \\ and
}

Mathematics Department

Stony Brook University

100 Nicolls Road

Stony Brook, NY 11794

U.S.A.

xiu@math.sunysb.edu 